\title{
Geometric effects in non-equilibrium electron transfer statistics in adiabatically driven quantum junctions
}

\author{
Himangshu Prabal Goswami ${ }^{1}$, Bijay Kumar Agarwalla ${ }^{2}$, and Upendra Harbola ${ }^{1}$ \\ ${ }^{1}$ Department of Inorganic and Physical Chemistry, \\ Indian Institute of Science, Bangalore-560012, India and \\ ${ }^{2}$ Chemical Physics Theory Group, Department of Chemistry, \\ and Centre for Quantum Information and Quantum Control, \\ University of Toronto, 80 Saint George St., Toronto, Ontario, Canada M5S 3 H6
}

(Dated: October 14, 2018)

\begin{abstract}
Cyclic Pancharatnam-Berry (PB) and adiabatic noncyclic geometric (ANG) effects are investigated in a single electron orbital system connected to two metal contacts with externally driven chemical potential and/or temperatures. The PB contribution doesn't affect the density matrix evolution, but has quantitative effect on the statistics (fluctuations) of electron transfer. The ANG contribution, on the other hand, affects the net flux across the junction. Unlike the PB, the ANG contribution is non-zero when two parameters are identically driven. Closed analytical expressions are derived for the ANG contribution to the flux, and the PB contribution to the first two leading order fluctuations. Fluctuations can be modified by manipulating the relative phases of the drivings. Interestingly, we find that the fluctuations of the pumped charge do not satisfy the steady state fluctuation theorem in presence of nonzero geometric contribution, but can be recovered for a vanishing geometric contribution even in presence of the external driving.

PACS numbers: 05.60.Gg,05.70.Ln,72.10.Bg,03.65.Vf
\end{abstract}

\section{INTRODUCTION}

A parametric modulation of a system Hamiltonian in an adiabatic fashion adds a phase change in system state. This phase results from holonomy of the parameter space and is known as the geometric phase $\mathbf{1}^{1.2}$. The geometric phase is quantified by the area traced in the parameter space. At least two independent parameters in the Hamiltonian should be subjected to time modulation. When the parametrization is cyclic, it is commonly referred to as the Pancharatnam-Berry (PB) phase ${ }^{3.4}$. For noncyclic evolution of the parameters, the acquired phase is known as adiabatic noncyclic geometric (ANG) phase $e^{5,6}$. The geometric phases realized in systems with no degeneracy in the eigenspace are usually referred to as abelian. In presence of degeneracy, the holonomies do not commute and give rise to non-abelian geometric phases $\underline{7-10}$.

Over the years, the effect of geometric phase has been studied in several systems such as solids $\frac{11}{1}$, condensed matter systems $\frac{12}{12}$ and quantum qubits 13 which can affect both the physical ${ }^{14}$ and chemical ${ }^{15}$ properties of quantum systems. There have been several attempts to understand the role of geometric phases in open quantum systems $\underline{16-21}$. However due to the complexity of non-equilibrium quantum systems, the exact role of geometric phase remains a mystery. A special type of non-equilibrium systems are quantum junctions where heat and electron transport are the key dynamic processes. These systems are made up of a quantum system which is coupled to reservoirs (bosonic or fermionic) at different thermodynamic states. Such junctions are realized in molecular break junctions ${ }^{22,23}$, quantum heat engines $\stackrel{24,25}{ }$, single molecules sandwiched be- tween a Scanning Tunneling Microscope (STM) tip and metal surface $\frac{26,27}{2}$ as well as single molecule electronic devices $28-30$. Most of the literature on quantum junctions focuses on the steady-state transport properties. In recent years it has been recognized that a time dependent probe (optical or electronic) coupled to transport measurements can lead to better characterization of the junction ${ }^{31-34}$. Such probes are usually fast (comparable to relaxation of the system). Here we explore the other extreme when the external driving is slow and the geometric phase is well defined. The geometric phase is known to create charge pumping in open quantum dots 35,36 and interference in spin currents through single-molecule magnets 37 . However its role in affecting electron transfer statistics has not been explored so far, although theoretical methods exist to explore the dynamics and statistics of such non-equilibrium quantum systems $\underline{38-43}$.

In this work, we study the geometric effects due to cyclic (PB) and noncyclic (ANG) adiabatic parametrization on electron transfer statistics induced by timedependent adiabatic change in thermodynamic equilibrium of electronic leads. We formulate a general theory of the geometric effects for non-equilibrium electron transport in weakly coupled systems and apply it to a single resonant level model. We find that the time-evolution of the reduced density matrix is not affected by the PB contribution but leads to a quantitative change in the statistics. On the other hand, the ANG part globally affects the dynamics. The PB contribution can be manipulated to alter the electron transfer statistics from antibunched to bunched by manipulating the phase-difference between the two drivings. It was recently shown that in case of bosonic reservoirs coupled to quantum system, pumping 
was possible by modulating the reservoir temperatures periodically 19 . We however find that for electron transfer, $\mathrm{PB}$ contribution does not affect the average electronic flux. The ANG part however affects the evolution of the density matrix and therefore contributes to the total electronic flux. In presence of geometric contributions (cyclic or noncyclic), fluctuations in the electrons exchanged between leads do not satisfy the standard fluctuation theorem $(\mathrm{FT}), \lim _{t \rightarrow \infty} \ln [P(q, t) / P(-q, t)]=q \mathfrak{F}^{\underline{43}}$, where $P(q, t)$ is the probability distribution function (PDF) for the net number $(q)$ of electrons exchanged between leads in a measurement time $t$, and $\mathfrak{F}$ is the thermodynamic force associated to the electron-flux. Similar violation of the fluctuation theorem or the Gallovoti-Cohen (GC) symmetry 44,45 was also reported in case of heat transport $^{19}$. However, unlike results of Ren et al ${ }^{19}$, for a vanishing geometric contribution, we recover the steady state FT for any phase difference between the drivings.

The paper is organized as follows. In Sec II we formulate a general interpretation of geometric contributions in weakly coupled quantum junction using Liouville space formalism 38 . In Sec. III, we present a driven quantum master equation (QME) for a single level system and analytically evaluate the $\mathrm{PB}$ contribution that arises due to a periodic time modulation of the thermodynamic equilibrium of the electronic reservoirs. In Sec. [IV] we discuss the effect of PB contribution in electron transfer statistics. In Sec $\nabla$ we show the violation of the steady-state fluctuation theorem, which is recovered for a vanishing geometric part. In Sec. VI we discuss the effect of ANG contribution on the dynamics of electron transport and then we conclude in Sec VII.

\section{THE GEOMETRIC CURVATURE}

Geometric effects can be realized in systems with adiabatic external driving. A weakly coupled system dynamics in the Liouville space is governed by the quantumLiouville equation ${ }^{46,47}$,

$$
|\dot{\rho}(t)\rangle\rangle=\hat{\mathcal{L}}(t)|\rho(t)\rangle\rangle,
$$

where $|\dot{\rho}(t)\rangle\rangle$ is the time rate of change of the reduced density vector for the system. $\hat{\mathcal{L}}(t)$ is the Liouvillian superoperator containing the time dependent driving. Equation (1) is valid for systems with no degenerate energy levels that get mixed due to the interaction with the baths (leads). The lead correlations decay much faster than the system relaxation. We assume that $\hat{\mathcal{L}}(t)$ is diagonalizable, has a single zero eigenvalue to guarantee the existence of a well defined steady state, and contains well-separated eigenvalues.

$$
\Lambda(t)=U^{-1}(t) \hat{\mathcal{L}}(t) U(t)
$$

Here, $U(t)\left(U^{-1}(t)\right)$ is a matrix composed of the instantaneous right (left) eigenvectors of $\hat{\mathcal{L}}(t)$ and diagonalizes
$\hat{\mathcal{L}}(t)$ to $\Lambda(t)$. This defines a new basis (eigenbasis of $\hat{\mathcal{L}}$ ), where

$$
\left.|\varrho(t)\rangle\rangle=U(t)^{-1}|\rho(t)\rangle\right\rangle .
$$

Equation of motion in this new basis is

$$
\left.|\dot{\varrho}(t)\rangle\rangle=\left[\Lambda(t)-U^{-1}(t) \dot{U}(t)\right]|\varrho(t)\rangle\right\rangle .
$$

In the adiabatic limit, the external driving is assumed to be much slower as compared to the internal system relaxations such that there are no transitions between the eigen states. This amounts to neglecting the offdiagonal terms of $U^{-1}(t) \dot{U}(t)$ in Eq.(41). We denote $B_{d}(t)=\operatorname{diag}\left\{U^{-1}(t) \dot{U}(t)\right\}$ and write the solution of Eq. (41) as

$$
\left.|\varrho(t)\rangle\rangle=e^{\int_{0}^{t} d t^{\prime} \Lambda\left(t^{\prime}\right)-\int_{0}^{t} d t^{\prime} B_{d}\left(t^{\prime}\right)}|\varrho(0)\rangle\right\rangle .
$$

The adiabatic approximation is considered only for the system evolution. The system evolution is adiabatic with respect its relaxation to the steady state determined by the coupling to the leads. There are three time scales: bath relaxation $\left(\tau_{B}\right)$, system relaxation $\left(\tau_{s}\right)$ due to coupling with the bath, and the time period $\left(t_{p}\right)$ of the external driving. The present formulation assumes that $\tau_{B} \ll \tau_{s} \ll t_{p}$. Faster the relaxation of the lead correlations, better is the approximation.

Using Eqs. (3) and (5), we get,

$$
\left.|\rho(t)\rangle\rangle=U(t) e^{\int_{0}^{t} d t^{\prime} \Lambda\left(t^{\prime}\right)} e^{-\int_{0}^{t} d t^{\prime} B_{d}\left(t^{\prime}\right)} U^{-1}(0)|\rho(0)\rangle\right\rangle .
$$

The first exponential is the usual 'dynamic' contribution to the time evolution. The second exponential is an additional part acquired due to the external driving and has a geometric interpretation. In the absence of driving, $B_{d}=0$. Let $\mathbf{x}$ represent a vector space corresponding to any two parameters, say $x$ and $y$, that are being modulated externally and periodically in time. We can then convert the time integral of the second exponential in Eq.(6) to a line integral along a contour $\mathcal{C}$, representing the instantaneous $x$ and $y$ values in the parameter space,

$$
\int_{0}^{t} d t^{\prime} B_{d}\left(t^{\prime}\right)=\int_{\mathcal{C}} d \mathbf{x} \cdot B_{d}(\mathbf{x}) .
$$

Equation (7) is a general expression valid for systems with adiabatic driving. If the time dependence is entirely due to the internal dynamics, i.e the system is prepared in the nonequilibrium state and evolves towards the steady state, then one cannot be sure that such an evolution will be adiabatic. In this case, separation between dynamic and geometric parts is not possible. So, Eq. (5) will not be a solution of Eq. (4). The externally controlled driving allows the evolution to be adiabatic such that Eq. (5) is valid and hence separation between dynamic and geometric parts is possible.

Assuming the contour in Eq. (6) to be closed (a fixed time period) and piecewise smooth, we can use Stokes' 
theorem and rewrite the contour integral as a surface integral over the surface $\mathcal{S}$ enclosed by the contour. This makes the factor geometric in nature. Note that, the dynamic part can not be given a geometric interpretation because there is no explicit time derivative which can be converted to a parametric integral. Equation (7) then becomes,

$$
\int_{\mathcal{C}} d \mathbf{x} \cdot B_{d}(\mathbf{x})=\oint_{\mathcal{C}} d \mathbf{x} \cdot B_{d}(\mathbf{x})=\oiint_{\mathcal{S}}\left(\nabla \times B_{d}(\mathbf{x})\right) \cdot d S .
$$

Here, $d S=d x d y$. The surface integrand $\nabla \times B_{d}(\mathbf{x})$ is equivalent to the geometric curvature where $B_{d}(\mathbf{x})$ represents the geometric vector potential. Note that, unlike the case of an isolated quantum dynamics of wavefunction, Eq. (8) cannot be interpreted as a phase factor because it affects the probability associated with an observable. Equation (8) represents a geometric contribution to the time evolution of the density matrix. In this reduced system dynamics, $\oiint_{\mathcal{S}}\left(\nabla \times B_{d}(\mathbf{x})\right)$. $d S$ is analogous to the Pancharatnam-Berry phase in isolated quantum dynamics and is not a phase as such, but is geometric in nature. This PB contribution is a direct manifestation of adiabatic and cyclic evolution of (at least) two parameters over a full time period of the drivings.

Equation (7) is a general expression for the acquired geometric contribution due to adiabatic modulation of two parameters. If the modulation is periodic, the contour $\mathcal{C}$ is closed in the parameter space and the resultant contribution, Eq. (8), is termed as the PB part. For nonperiodic driving $\mathcal{C}$ is an open contour with a geometric interpretation, called the ANG part, which we shall discuss in section VI.

\section{MODEL CALCULATION}

We consider a single electronic orbital coupled to two electronic reservoirs kept at different chemical potentials as shown in Fig.(11). The Hamiltonian for this system is given by

$$
\begin{aligned}
\hat{H} & =\hat{H}_{s}+\hat{H}_{l}+\hat{H}_{r}+\hat{H}_{s \nu} \\
& =\epsilon_{s} \hat{c}_{s}^{\dagger} \hat{c}_{s}+\sum_{l} \epsilon_{l} \hat{c}_{l}^{\dagger} \hat{c}_{l}+\sum_{r} \epsilon_{r} \hat{c}_{r}^{\dagger} \hat{c}_{r} \\
& +\sum_{\nu \in l, r}\left(T_{s \nu} \hat{c}_{s}^{\dagger} \hat{c}_{\nu}+\text { h.c. }\right),
\end{aligned}
$$

where $s, l, r$, are the system, the right and the left lead orbitals, respectively. $\hat{H}_{s \nu}$ is the system-lead coupling Hamiltonian such that $T_{s \nu}$ is the coupling between the system and leads with $\nu=l, r$, and $\hat{c}_{s}^{\dagger}\left(\hat{c}_{s}\right), \hat{c}_{l}^{\dagger}\left(\hat{c}_{l}\right)$ and $\hat{c}_{r}^{\dagger}\left(\hat{c}_{r}\right)$ represent the electronic creation(annihilation) operators for the system, left and right leads, respectively. The leads have no interactions and are always in equilibrium (they act as reservoirs) and exchange spinless charges with the system.

We consider the case when the thermodynamic equilibrium of the electronic reservoirs is modulated in time

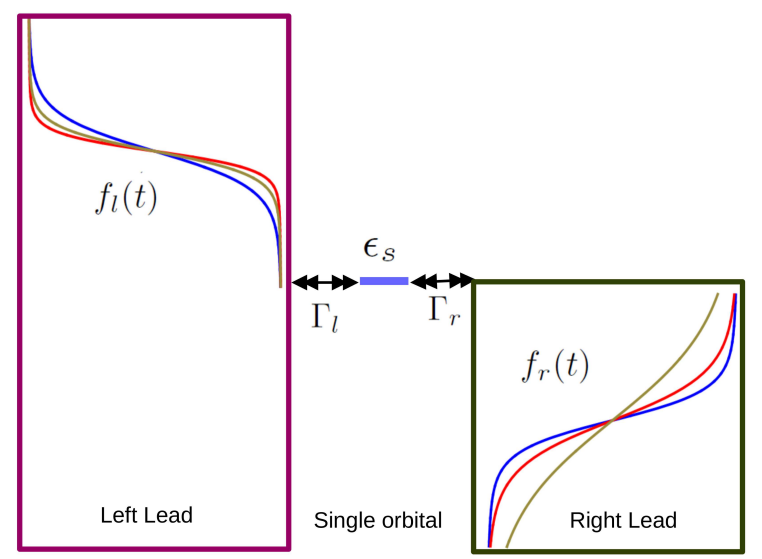

FIG. 1. Schematics of the driven single resonant level model. The leads are metallic and spinless charge transfer occurs between the single orbital and leads. The Fermi distributions of the metal leads in resonance with the orbital's energy, $\epsilon_{s}$, are driven periodically in time. $\Gamma_{l(r)}$ is the coupling of the orbital with the left (right) lead.

by altering the temperature or the chemical potential. A possible way to experimentally visualize the driven quantum junction would be to connect the metal contacts (electronic reservoirs) to a time varying thermostat. This would allow control on the Fermi-functions of the metal leads through the time dependent temperatures. Or one may use a time varying gate-voltage to modulate energy of the orbital or use voltage pulses to control chemical potential of the leads. All these in turn will change the Fermi distribution of the leads.

The driven quantum master equation for the reduced system dynamics is given by,

$$
\begin{aligned}
\dot{\rho}(t) & =\left[\Gamma_{l} \tilde{f}_{l}(t)+\Gamma_{r} \tilde{f}_{r}(t)\right]\left[\hat{c}_{s} \rho(t) \hat{c}_{s}^{\dagger}-\hat{c}_{s}^{\dagger} \hat{c}_{s} \rho(t)\right] \\
& +\left[\Gamma_{l} f_{l}(t)+\Gamma_{r} f_{r}(t)\right]\left[\hat{c}_{s}^{\dagger} \rho(t) \hat{c}_{s}-\rho(t) \hat{c}_{s} \hat{c}_{s}^{\dagger}\right] .
\end{aligned}
$$

Here, $\Gamma_{X}=\pi n_{X}\left(\epsilon_{s}\right)\left|T_{s X}\right|^{2}$ with $n_{X}$ representing the density of states of lead $X$. The adiabatic limit is valid for $t_{p}\left(\Gamma_{l}+\Gamma_{r}\right) \gg 1$ and $\tau_{s} \approx\left(\Gamma_{l}+\Gamma_{r}\right)^{-1}$. The time dependent Fermi-functions for the leads is $f_{X}(t)=$ $\left(e^{\bar{\beta}_{X}\left(\epsilon_{s}-\mu_{X}\right)}+1\right)^{-1}$, and $\tilde{f}_{X}(t)=1-f_{X}(t) . \quad \mu_{X}$ and $\bar{\beta}_{X}$ is the chemical potential and inverse temperature of the $X \in l, r$-th lead, respectively. The Fermi-functions depend on both temperature and the chemical potential. Changing either of these inherently affects the Fermi functions of the leads. So we choose the driving protocol in terms of the Fermi distributions for simplicity. This implicit dependence on time either via the inverse temperatures $\bar{\beta}_{X}(t)=\left(k_{B} T(t)\right)^{-1}$ or the chemical potential of the $X$ th lead, $\mu_{X} \rightarrow \mu_{X}(t)$ is arbitrary as long as the bath correlations die fast. For the model considered here, the density vector contains only two elements $\rho_{11}$ and $\rho_{00}$ representing the population of the many-body state with 1 and 0 electrons, respectively. Coherences do not couple to populations and die off exponentially. Since we are interested in the steady-state dynamics (adiabatic driving), coherences will be ignored. We define 
$|\rho(t)\rangle\rangle=\left\{\rho_{11}, \rho_{00}\right\}$. The time dependent Liouvillian in Eq. (11) is then,

$$
\hat{\mathcal{L}}(t)=2\left(\begin{array}{cc}
-\alpha(t) & \beta(t) \\
\alpha(t) & -\beta(t)
\end{array}\right)
$$

where $\alpha(t)=\Gamma_{l} \tilde{f}_{l}(t)+\Gamma_{r} \tilde{f}_{r}(t)$ and $\beta(t)=\Gamma_{l} f_{l}(t)+$ $\Gamma_{r} f_{r}(t)$ are the system to leads and leads to system electron transfer rates, respectively.

As we shall discuss below, for the single resonant level case that we consider here, when the Fermi functions are adiabatically modulated (i.e the parameter space, $\mathbf{x}$ is composed of the time-dependent parameters $f_{l}(t)$ and $\left.f_{r}(t)\right)$, the vector potential, $B_{d}\left(f_{l}, f_{r}\right)$ is non-zero. However, the overall $\mathrm{PB}$ contribution vanishes (SecVI). This happens because the curvature is zero, $\nabla \times B_{d}\left(f_{l}, f_{r}\right)=0$, as a result of the periodic driving. So, $B_{d}\left(f_{l}, f_{r}\right)$ is a conservative or irrotational field in the parameter space. Thus, for a two parameter periodic and adiabatic evolution, the PB contribution doesn't affect the dynamics of the reduced density-matrix of the single resonant level, although it does influence the statistics of the electron transfer between the system and leads.

\section{ELECTRON TRANSFER STATISTICS}

To quantify the effect of PB contribution on the statistics of electron transfer, we consider probability distribution function, $P(q, t)$ for the net, $q$, number of electrons transferred between system and leads. We define a generating function $G(\lambda, t)$ corresponding to $P(q, t) \underline{43}$

$$
G(\lambda, t)=\sum_{q} P(q, t) e^{\lambda q} \equiv\langle\langle\mathbf{1} \mid \rho(\lambda, t)\rangle\rangle .
$$

Here $|\mathbf{1}\rangle\rangle$ is the identity vector and $|\rho(\lambda, t\rangle\rangle$ is the $\lambda$ dependent density vector obeying the equation of motion (appendix),

$$
|\dot{\rho}(\lambda, t)\rangle\rangle=\hat{M}(\lambda, t)|\rho(\lambda, t)\rangle\rangle .
$$

$\hat{M}(\lambda, t)$ is the $\lambda$-dependent Liouvillian (appendix) given by

$$
\hat{M}(\lambda, t)=2\left(\begin{array}{cc}
-\alpha(t) & \beta_{l}(t) e^{\lambda}+\beta_{r}(t) \\
\alpha_{l}(t) e^{-\lambda}+\alpha_{r}(t) & -\beta(t)
\end{array}\right) .
$$

Here $\alpha_{X}=\Gamma_{X} \tilde{f}_{X}(t)$ and $\beta_{X}=\Gamma_{X} f_{X}(t), X \in l, r$. For $\lambda=0$, Eq. (13) reduces to Eq. (11) and $|\rho(\lambda=0, t)\rangle\rangle=$ $|\rho(t)\rangle\rangle$.

Since we are interested in the steady state fluctuations, we define a scaled cumulant generating function 43,48 ,

$$
S(\lambda)=\lim _{t \rightarrow \infty} \frac{1}{t} \ln G(\lambda, t) .
$$

In the long time limit, it can be shown that scaled cumulant generating function is additively separable into two parts 19,49 (appendix), viz. dynamic, $S_{d}(\lambda)$, and a geometric, $\left.S_{g}(\lambda)\right)$; i.e $S(\lambda, t)=S_{d}(\lambda, t)+S_{g}(\lambda, t)$ where,

$$
\begin{aligned}
& S_{d}(\lambda)=\frac{1}{t_{p}} \int_{0}^{t_{p}} d t^{\prime} \zeta_{+}\left(\lambda, t^{\prime}\right), \\
& S_{g}(\lambda)=\frac{-1}{t_{p}} \int_{0}^{t_{p}} d t^{\prime}\left\langle\left\langle L_{+}\left(\lambda, t^{\prime}\right)\left|\frac{\partial}{\partial t^{\prime}}\right| R_{+}\left(\lambda, t^{\prime}\right)\right\rangle\right\rangle .
\end{aligned}
$$

$\left.\left|R_{+}\left(\lambda, t^{\prime}\right)\right\rangle\right\rangle\left[\left\langle\left\langle L_{+}\left(\lambda, t^{\prime}\right)\right|\right]\right.$ denote the instantaneous right [left] eigenvectors of $\hat{M}\left(\lambda, t^{\prime}\right)$ corresponding to the instantaneous smaller eigenvalue $\zeta_{+}\left(\lambda, t^{\prime}\right)$. The eigenvalues of $\hat{M}(\lambda, t)$ in Eq. (14) are,

$$
\zeta_{ \pm}(\lambda, t)=-\Gamma \pm \sqrt{\Gamma^{2}+4\left(\alpha_{r}(t) \beta_{l}(t)\left(e^{\lambda}-1\right)+\alpha_{l}(t) \beta_{r}(t)\left(e^{-\lambda}-1\right)\right)}
$$

Here $\Gamma=\Gamma_{l}+\Gamma_{r}$. Also, the measurement time, $t=$ $\nu t_{p}$, where $\nu$ is the number of cycles and $t_{p}$ is the timeperiod of the driving such that $\Gamma t_{p} \gg 1$. We can write $S_{g}$ as a line integral over a closed contour $\mathcal{C}$ defined in the parameter space such that,

$$
S_{g}(\lambda)=\frac{-1}{t_{p}} \oint_{\mathcal{C}} d \mathbf{x} \cdot\left\langle\left\langle L_{+}(\lambda, \mathbf{x})\left|\partial_{\mathbf{x}}\right| R_{+}(\lambda, \mathbf{x})\right\rangle\right\rangle .
$$

Here, vector $\mathbf{x}$ contains system parameters modulated by the external driving. For $\lambda=0$, the integrand in Eq. (19) is equivalent to $B_{d}(\mathbf{x})$ in Eq. (6). We can now convert the line integral to a surface integral over the contour area, $\mathcal{S}$, for a closed $\mathcal{C}$. Equation (19), can be recast as,

$$
S_{g}(\lambda)=\frac{1}{t_{p}} \oiint_{\mathcal{S}} d x d y \mathfrak{B}^{\lambda}(x, y)
$$

with $\mathfrak{B}^{\lambda}(x, y)=\mathfrak{B}_{x y}^{\lambda}-\mathfrak{B}_{y x}^{\lambda}$ being equivalent to the Pancharatnam-Berry curvature in the parametric space of $x$ and $y$ (appendix), where

$$
\mathfrak{B}_{x y}^{\lambda}=\frac{\left\langle\left\langle L\left(\zeta_{-}\right)\left|\partial_{x} \hat{M}(\lambda)\right| R\left(\zeta_{+}\right)\right\rangle\right\rangle\left\langle\left\langle L\left(\zeta_{+}\right)\left|\partial_{y} \hat{M}(\lambda)\right| R\left(\zeta_{-}\right)\right\rangle\right\rangle}{-\left(\zeta_{+}-\zeta_{-}\right)^{2}} .
$$

When $\lambda=0, \mathfrak{B}^{\lambda=0}(x, y) \equiv \nabla \times B_{d}(x, y)$. Note that, the Pancharatnam-Berry curvature (Eq. (21) ) is identically zero if only a single parameter is changed in periodic manner. This is because $\mathfrak{B}^{\lambda}(x, y)=0$. If two mutually dependent parameters are changed, $\mathfrak{B}_{x y}^{\lambda}=\mathfrak{B}_{y x}^{\lambda}$, resulting in vanishing curvature, $\mathfrak{B}^{\lambda}(x, y)=0$.

Here we choose to modulate the Fermi functions of the leads, i.e., $x=f_{l}(t), y=f_{r}(t)$. The $\lambda$-dependent $\mathrm{PB}$ curvature, $\mathfrak{B}^{\lambda}\left(f_{l}, f_{r}\right)$, simplifies to,

$$
\mathfrak{B}^{\lambda}\left(f_{l}, f_{r}\right)=\frac{e^{-\lambda}\left(e^{\lambda}-1\right)^{2} \Gamma_{l} \Gamma_{r}\left(\Gamma_{l}-\Gamma_{r}\right)}{\left\{\Gamma_{l}^{2}+\Gamma_{r}^{2}+2 \Gamma_{l} \Gamma_{r}\left(\tilde{f}_{l}-f_{l}\right)\left(\tilde{f}_{r}-f_{r}\right)+\mathfrak{Z}_{\lambda}\right\}^{\frac{3}{2}}}
$$

with

$$
\mathfrak{Z}_{\lambda}=4 \Gamma_{l} \Gamma_{r}\left(f_{l} \tilde{f}_{r} e^{\lambda}+f_{r} \tilde{f}_{l} e^{-\lambda}\right) .
$$

When, $\Gamma_{l}=\Gamma_{r}$, the PB curvature in Eq. (22) is zero. This happens because $\left\langle\left\langle L_{+}\left(\lambda, t^{\prime}\right) \mid \dot{R}_{+}\left(\lambda, t^{\prime}\right)\right\rangle\right\rangle$ in Eq. (17), 
becomes a total time derivative which integrated over the time period $t_{p}$ becomes zero. So, under a symmetric coupling, $\Gamma_{l}=\Gamma_{r}$, the Pancharatnam-Berry contribution is zero and the statistics is governed solely by the dynamic part, $S_{d}(\lambda)$. Below we shall always consider the case when $\Gamma_{l} \neq \Gamma_{r}$, so that we have a finite geometric (PB) contribution. From Eq. (21), we also note that when $\lambda=0, \mathfrak{B}^{\lambda=0}\left(f_{l}, f_{r}\right)=0$. That is the dynamics of the reduced density matrix of the single resonant level is not affected by the geometric (PB) contribution. However the statistics of electron transfer is influenced by the geometric part, as we discuss below.

We focus on the $i$ th cumulants of the net probability distribution function $P(q, t)$ which are obtained from the $i$ th $\lambda$-derivatives of the scaled cumulant generating function,

$$
C^{(i)}=\left.\frac{d^{i}}{d \lambda^{i}} S_{d}(\lambda)\right|_{\lambda=0}+\left.\frac{d^{i}}{d \lambda^{i}} S_{g}(\lambda)\right|_{\lambda=0} .
$$

Equation (22) can be substituted in Eq. (20) to compute $S_{g}(\lambda)$ from which the geometric correction to cumulants, $\left.\frac{d^{i}}{d \lambda^{i}} S_{g}(\lambda)\right|_{\lambda=0}$, can be evaluated. We find that the correction to the first cumulant (average flux) is zero as reported earlier ${ }^{50}$. The PB contribution has no effect on the average electronic flux between the system and leads.

$$
C_{g}^{(1)}=\frac{1}{t_{p}} \oiint_{\mathcal{S}}\left(\left.\frac{d}{d \lambda} \mathfrak{B}^{\lambda}\left(f_{l}, f_{r}\right)\right|_{\lambda=0}\right) d f_{l} d f_{r}=0 .
$$

Note that the average flux is $j=\operatorname{Tr}\{\hat{I} \rho(t)\}$, where $\hat{I}$ is current operator. Since $|\rho(t)\rangle\rangle$ doesn't have a PB part, $j$ is also independent of it. In fact, the expectation value of all single time observables will be unaffected from the PB contribution. The PB part, however, contributes to higher cumulants through higher order correlation functions of time dependent observables. The contributions to fluctuation (second cumulant) and skewness (third cu- mulant) from the geometric parts are calculated as,

$$
\begin{aligned}
C_{g}^{(2)} & =\left.\frac{1}{t_{p}} \oiint_{\mathcal{S}} \frac{d^{2}}{d \lambda^{2}} \mathfrak{B}^{\lambda}\left(f_{l}, f_{r}\right)\right|_{\lambda=0} d f_{l} d f_{r} \\
& =\frac{1}{t_{p}} \frac{2 C_{A} \Gamma_{l} \Gamma_{r}\left(\Gamma_{l}-\Gamma_{r}\right)}{\left(\Gamma_{l}+\Gamma_{r}\right)^{3}}, \\
C_{g}^{(3)} & =\frac{1}{t_{p}} \oiint_{\mathcal{S}}\left(\left.\frac{d^{3}}{d \lambda^{3}} \mathfrak{B}^{\lambda}\left(f_{l}, f_{r}\right)\right|_{\lambda=0} d f_{l} d f_{r}\right) \\
& =\frac{1}{t_{p}} \frac{36 \Gamma_{l}^{2} \Gamma_{r}^{2}\left(\Gamma_{l}-\Gamma_{r}\right)}{\left(\Gamma_{l}+\Gamma_{r}\right)^{5}} \oiint_{\mathcal{S}}\left(f_{l}-f_{r}\right) d f_{l} d f_{r} .
\end{aligned}
$$

Here $C_{A}=\oiint_{\mathcal{S}} d f_{l} d f_{r}$ is the contour area in the parameter space of $f_{l}$ and $f_{r}, 0 \leq f_{l}, f_{r} \leq 1$. We give analytical expressions of $C_{A}$ for a sinusoidal driving in the appendix. Therefore, the PB contribution has a quantitative effect on the statistics of electron transfer through the second and higher order cumulants. The PB corrections to the second and third cumulants as given in Eqs. (27) and (29), can be positive or negative depending on the relative values of $\Gamma_{l}$ and $\Gamma_{r}$.

The statistics of electron transfer is usually quantified using the Fano-factor $(F)^{\underline{51}-\underline{53}}$ and is defined as the ratio between the second and first cumulants. When $F>1(F<1)$, the transferred electrons between system and leads are correlated (anti-correlated) and gives rise to bunched 54,55 (antibunched, 56,57 ) statistics. In the present case, $F$ is obtained as,

$$
\begin{aligned}
F & =\frac{C_{d}^{(2)}+C_{g}^{(2)}}{C_{d}^{(1)}} \\
& =\frac{C_{d}^{(2)}}{C_{d}^{(1)}}+\frac{2 C_{A} \Gamma_{l} \Gamma_{r}\left(\Gamma_{l}-\Gamma_{r}\right)}{t_{p}\left(\Gamma_{l}+\Gamma_{r}\right)^{3} C_{d}^{(1)}},
\end{aligned}
$$

where the first term is due to the dynamic part with

$$
\begin{aligned}
C_{d}^{(1)} & =\frac{1}{t_{p}} \int_{0}^{t_{p}} d t \frac{\left.2\left[\alpha_{r}(t) \beta_{l}(t)-\alpha_{l}(t) \beta_{r}(t)\right)\right]}{\sqrt{(\alpha(t)-\beta(t))^{2}+4\left(\alpha_{r}(t) \beta_{l}(t)+\alpha_{l}(t) \beta_{r}(t)\right)}} \\
C_{d}^{(2)} & =\frac{2}{t_{p}} \int_{0}^{t_{p}} d t \frac{\left(\alpha_{r}(t) \beta_{l}(t)\left((\alpha(t)-\beta(t))^{2}+2 \alpha_{r}(t) \beta_{l}(t)\right)+\alpha_{l}(t) \beta_{r}(t)\left[(\alpha(t)-\beta(t))^{2}+6 \alpha_{r}(t) \beta_{l}(t)\right]+4 \alpha_{l}^{2}(t) \beta_{r}^{2}(t)\right)}{\sqrt{\left((\alpha(t)-\beta(t))^{2}+4\left(\alpha_{r}(t) \beta_{l}(t)+\alpha_{l}(t) \beta_{r}(t)\right)\right)}}
\end{aligned}
$$

Since $C_{d}^{(2)}$ is symmetric with respect to interchange of $l$ and $r$, while $C_{g}^{(2)}$ is antisymmetric and can be positive, negative, or zero, depending on the relative values of $\Gamma_{l}$ and $\Gamma_{r}$, the statistics of the net electron transfer can be changed by tuning the fluctuations via $\mathrm{PB}$ contribution alone. Choosing $\Gamma_{l}>\Gamma_{r}\left(\Gamma_{l}<\Gamma_{r}\right)$, the PB part enhances (suppresses) the fluctuations. This effect is shown in Fig.(2) for sinusoidal drivings: $f_{l}(t)=$ $f_{l}\left(1-m^{2} \cos ^{2}(k t)\right), f_{r}(t)=f_{r}\left(1-m^{2} \cos ^{2}(k t+\phi)\right)$. Here, $k=\pi / t_{p}$ is the driving frequency, $\phi$ is the phase difference between the two drivings and $0 \leq m<1$. Here, the quantities $f_{l}$ and $f_{r}$ are the Fermi functions of the left and the right leads respectively, evaluated at the energy $\epsilon_{s}$, in the absence of driving $(m=0)$. As shown in Fig. (2), the Fano factor can be increased beyond unity by tuning $\Gamma_{r}$ or by changing the phase difference between the two drivings. For $\phi=0$, the PB contribution is zero 


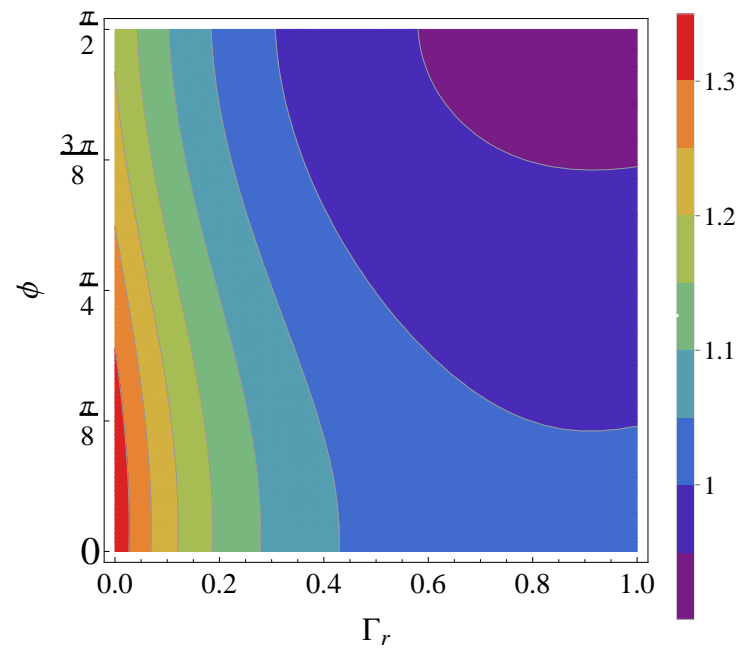

FIG. 2. The Fano factor $(F)$ contour plot for $\Gamma_{r}$ and $\phi$. The PB contribution is introduced by changing the Fermifunctions as $f_{l}(t)=f_{l}\left(1-m^{2} \cos ^{2}(k t)\right), f_{r}(t)=f_{r}(1-$ $\left.m^{2} \cos ^{2}(k t+\phi)\right) . \quad k=\pi / t_{p}$ is the driving frequency, $t_{p}$ is the driving time period, $\phi$ is the phase difference between the two drivings and $0<m<1$. Simulation parameters are $m=0.9, k=1, \nu=10, f_{l}=0.9, f_{r}=0.2 . \Gamma_{l}=0.9$. Second curve from the top-right represent the boundary between $F>1$ and $F<1$.

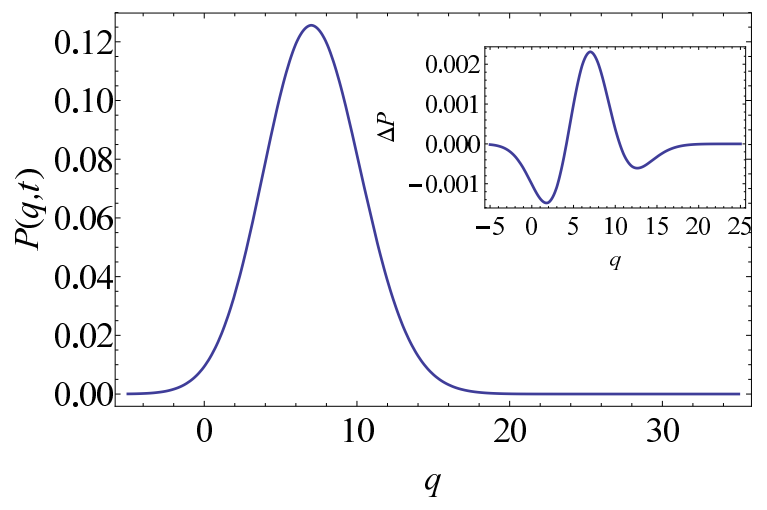

FIG. 3. The net interpolated PDF in presence of PB contribution $(\phi \neq 0)$, simulated at $f_{l}=0.75, f_{r}=0.25, k=1, \nu=$ $20, k=1, m=0.9, \phi=\frac{\pi}{4}, \Gamma_{r}=0.25$ and $\Gamma_{l}=1$. The inset shows the difference between the PDFs in presence and absence of $\mathrm{PB}$ part.

(appendix). For nonzero $\phi$, the statistics is bunched for small values of $\Gamma_{r}$ and tends to become antibunched as $\Gamma_{r}$ is increased. Over a range of small values of $\phi$, statistics is always bunched.

\section{STEADY-STATE FLUCTUATION THEOREM}

For a non-driven case, it is known that the generating function satisfies a linear symmetry, $G(\lambda)=G(-\lambda-\mathfrak{F})^{\underline{43}}$, where $\mathfrak{F}$ is the thermodynamic affinity (nonequilibrium force). This is reflected in the fluctuation symmetry, also referred to as GC symmetry, in $P(q, t)$ as $\stackrel{43}{ }$,

$$
\lim _{t \rightarrow \infty} \ln \frac{P(q, t)}{P(-q, t)}=q \mathfrak{F},
$$

where $\mathfrak{F}=\ln \left\{f_{r}\left(1-f_{l}\right) / f_{l}\left(1-f_{r}\right)\right\}$ for the resonant level model.

For the driven case, the full probability distribution function is computed by inverting Eq. (12) after analytic continuation,

$$
P(q, t)=\frac{1}{2 \pi} \int_{0}^{2 \pi} d \lambda G(i \lambda, t) e^{-i \lambda q} .
$$

We evaluate Eq. (35) numerically for a fixed measurement time $t=\nu t_{p}$. In Fig.(3), we compare the distributions in presence and in absence of $\mathrm{PB}$ contribution. The mean of both the distributions is the same but the fluctuations are different. This is highlighted in the inset of Fig.(3), which shows the difference in the values of the PDFs in presence and absence of $\mathrm{PB}$ part, $\Delta P=P_{o}(q, t)-P(q, t)$, where $P_{o}(q, t)$ is the PDF without the PB contribution. Since the average flux is independent of $\mathrm{PB}, \int q \Delta P d q=0$, also due to normalization of $P(q, t)$ and $P_{o}(q, t), \int \Delta P d q=0$.

The distribution, $P(q, t)$, can also be evaluated using the Gartner-Ellis/Varadhan theorem ${ }^{58}$, valid at large measurement times, where we can write,

$$
P(y) \approx N(t) e^{-t \mathfrak{L}(y)}
$$

where, $y=q / t$ is the rate of electron transfer and $N(t)$ is a time dependent normalization constant. The " $\approx$ "sign indicates that the result is valid only at long times. $\mathfrak{L}(y)$ is the Legendre-Fenchel transformation of the scaled cumulant generating function known as the large deviation function 58,59 (LDF) defined as,

$$
\mathfrak{L}(y)=\operatorname{ext}_{\lambda}(y \lambda-S(\lambda)),
$$

where ext represents the extremum value(supremum or infimum). The full cumulant generating function for the driven case, $S(\lambda)$, is obtained by combining Eqs. (16) and (20), where $\zeta_{+}(\lambda, t)$ and $\mathfrak{B}^{\lambda}\left(f_{l}, f_{r}\right)$ are given by Eqs. (18) and (21), respectively. The large deviation method is based on the saddle point approximation ${ }^{60}$ and is reliable only when Eq. (37) is strictly convex or concave and a well defined extremum value for $\lambda$ exists 58 .

Using Eq. (15), in the long time limit, Eq. (35) can be written as,

$$
P(q, t) \approx \frac{1}{2 \pi} \int_{0}^{2 \pi} d \lambda e^{-i \lambda q+t S(i \lambda)} .
$$

We expand the function, $i \lambda q-t S(i \lambda)$ in Eq. (38) around its extremum value $\lambda_{q}^{*}$ (the saddle point), and retain the first two leading order terms to get,

$$
P(q, t) \approx e^{-t \mathfrak{L}(y)} T^{(2)}
$$




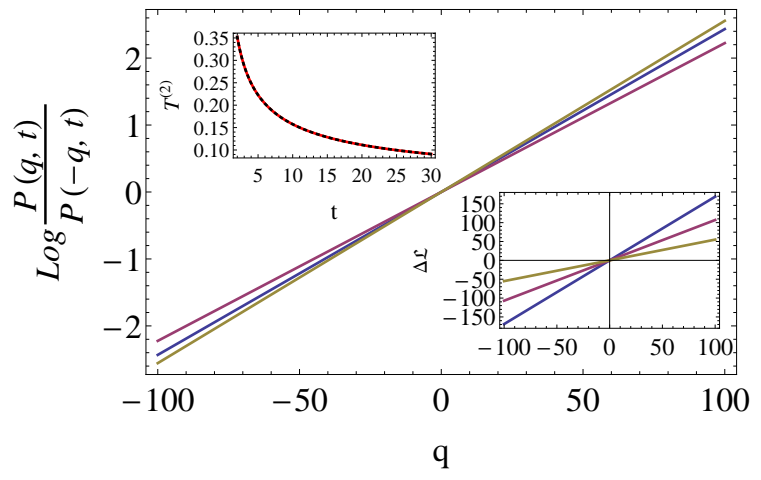

FIG. 4. The steady state driven fluctuation theorem, Eq.(44). All curves are simulated at $\Gamma_{l}=\Gamma_{r}=0.25, f_{l}=$ $0.75, f_{r}=0.25, k=1, \nu=20, k=1, m=0.9$. The three curves with different slopes $(R)$ correspond to $\phi=0(R=$ 1.38), $\frac{\pi}{3}(1.61), \frac{\pi}{4}(1.53)$. The bottom-right inset shows results for $\Delta \mathfrak{L}$, Eq. (42), at $\phi=0, \Gamma_{l}=0.75, \Gamma_{r}=0.25$. As $f_{l}$ increases the slope $(R)$ increases, $f_{l}=0.9(R=$ $1.69), 0.6(1.07), 0.4(0.55)$. All other parameters are the same as in the main figure. The top left inset represents the decay of Eq. (40), fitted with a power law: $t^{-0.49}$ for $q=9$, $\phi=\pi / 4, m=0.2, \Gamma_{l}=0.75$ and $\Gamma_{r}=0.25$ The time axis has been rescaled by a factor of $10^{-2}$.

where,

$T^{(2)}=\frac{1}{2 \pi} \int_{0}^{2 \pi} d \lambda \exp \left\{\frac{-t}{2 t_{p}} \int_{0}^{t_{p}} d \tau \frac{d^{2} \zeta_{+}(i \lambda, \tau)}{d(i \lambda)^{2}}\left(i \lambda-\lambda_{q}^{*}\right)^{2}\right\}$

We evaluate the term $T^{(2)}$ numerically and find that it asymptotically goes to zero as a power law $1 / \sqrt{t}$, as shown in the top left inset of Fig. (41). At long times, $(1 / t) \log T^{(2)}$ therefore, decays as an inverse power law, $1 / t$. Thus, at large measurement times, the cumulant generating function can be approximated using the large deviation result, Eq. (36).

In the case of heat transfer between two thermal baths, it was reported by Ren et al $\frac{19}{19}$, that the scaled cumulant generating function does not satisfy the usual symmetry, $S(\lambda)=S(-\lambda-\mathfrak{F})$, even in the absence of the PB contribution. In the present case, however, we find that such a symmetry is preserved, as we discuss below.

The LDF obtained from Eq. (37) is of the form,

$$
\mathfrak{L}(q, t)=\frac{q}{t} \lambda_{q}^{*}-S\left(\lambda_{q}^{*}\right),
$$

where $\lambda_{q}^{*}$ is the value of $\lambda$ at fixed time $t$ which satisfies the RHS of Eq.(37). We numerically evaluate Eq. (41) for the case when the PB contribution is zero which can be achieved by choosing either $\phi=0$ or $\Gamma_{l}=\Gamma_{r}$. We find that the following equality is satisfied,

$$
\Delta \mathfrak{L}=\mathfrak{L}(-q, t)-\mathfrak{L}(q, t)=q \frac{R}{t},
$$

where,

$$
R=\ln \frac{\int_{0}^{t_{p}} d \tau f_{r}(\tau)\left(1-f_{l}(\tau)\right)}{\int_{0}^{t_{p}} d \tau f_{l}(\tau)\left(1-f_{r}(\tau)\right)},
$$

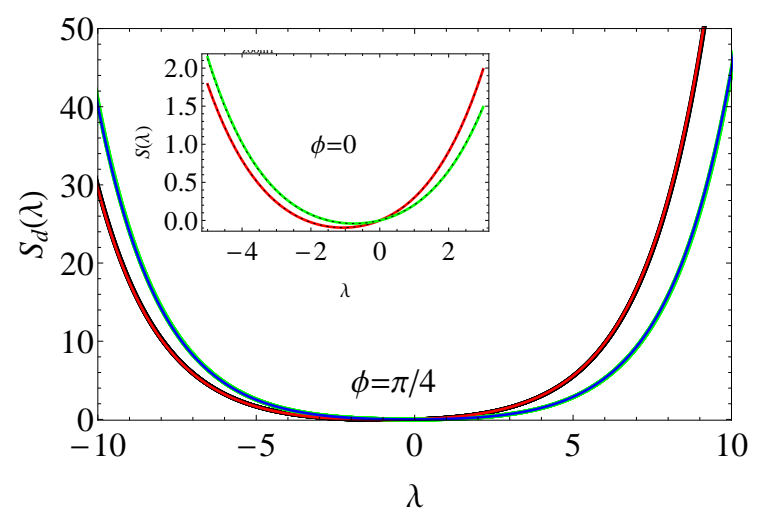

FIG. 5. The dynamic cumulant generating function, $S_{d}(\lambda)$ (solid) and linearly shifted, $S_{d}(-\lambda-R)$ (dotted). The curves are indistinguishable due to the GC symmetry, $S_{d}(\lambda)=$ $S_{d}(-\lambda-R)$ where, $R$ is the thermodynamic force given in Eq. (43). All curves are simulated at $\Gamma_{l}=0.75, \Gamma_{r}=$ $0.25, f_{l}=0.75, f_{r}=0.25, k=1, \nu=20, k=1, \phi=\pi / 4$. The two curves, red, blue are evaluated for $m=0.2(R=$ $2.153), 0.5(1.92)$ respectively. The inset is evaluated at $\phi=$ $0, \Gamma_{l}=0.75, \Gamma_{r}=0.25$. The two curves (red, green) represent $m=0.2(2.145), 0.5(1.9)$ respectively.

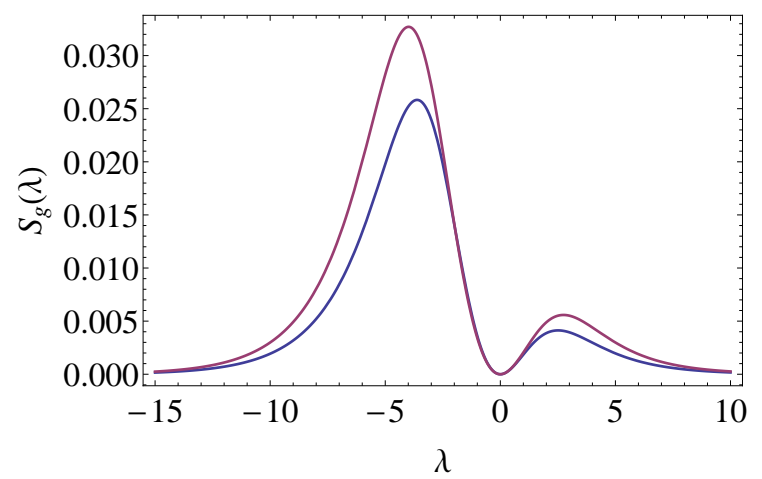

FIG. 6. The asymmetric geometric cumulant generating function. $\Gamma_{l}=1$ (higher peak), 0.75 (lower peak). All other parameters are same as in Fig. (5). Because of the asymmetric behavior of $S_{g}(\lambda)$, the linear symmetry, $S(\lambda)=S(-\lambda-R)$, does not hold in the presence of the geometric contribution, resulting in violation of the FT, Eq. (44).

which reduces to $\mathfrak{F}$ (Eq. (34)) in absence of the drivings. Although Eq. (42) is numerically verified (bottom-right inset of Fig. (4) $)$, its validity can be justified. When $R=0, \Delta \mathfrak{L}=0$, giving $P(q, t)=P(-q, t)$ at long time, i.e equilibrium is attained. As the system moves out of equilibrium, $R$ in non-zero and a flux develops in the system. The average flux is given below in Eq. (62) where $R$ is analytically identified as the thermodynamic force. For the non-driven case, Eq. (42) is always valid and $R \rightarrow \mathfrak{F}$. $\mathfrak{F}$ is the thermodynamic force for the non driven case. So the basic structure of Eq. (42) for the driven (without geometric part) and non-driven case remain the same. Thus identification of the thermodynamic force by $R$ as given in Eq. (43) is consistent with the definition 
of equilibrium $(P(q, t)=P(-q, t))$ and the flux, both for the driven (without the geometric part) and non-driven system.

Equations (42) and (43) together lead to a driven steady-state fluctuation theorem of the type $\underline{\underline{\underline{61}} \text {, }}$

$$
\lim _{t \rightarrow \infty} \frac{P(q, t)}{P(-q, t)}=e^{q R}
$$

preserving the GC type of symmetry in the absence of geometric term. As long as $f_{l}>f_{r}, R$ is a positive quantity and is the thermodynamic force driving the flux in the system. In Figs. (4) and (5), we show the validity of the GC symmetry and the steady-state driven fluctuation theorem in absence of the geometric term . In Fig. (5), we show the symmetry, $S(\lambda)=S(-\lambda-R)$, where $R$ is defined by Eq. (43).

For the case when the geometric contribution is nonzero, $\mathfrak{B}^{\lambda}\left(f_{l}, f_{r}\right) \neq 0$, Eqs. (42) and (44) are not valid. This is because $S_{g}(\lambda)$ does not possess the same linear (translational) symmetry as the dynamic part, $S_{d}(\lambda)=S_{d}(-\lambda-R)$. Lack of this symmetry in presence of the PB part results in the breaking of the steady state FT. We further observe that the symmetry is broken only near low values of $\lambda$ where the effect of PB is most prominent as shown in Fig. (6). As $\lambda$ increases $S_{g}(\lambda)$ goes to zero and the linear symmetry in $S(\lambda)$ is recovered for large $\lambda$, implying that, in the limit of large $q$, the FT will be violated, however Eqs. (42) and (44) should be recovered for small $q$ values.

Note that, adiabatic drivings with $\phi \neq 0$ give rise to geometric contributions that results in the break-down of FT. However, it is to be emphasized that mere presence of the phase-different drivings doesn't violate the FT, since the geometric contribution may still be zero. For example, in the present case, the geometric contribution is zero for all $\phi$ if $\Gamma_{l}=\Gamma_{r}$, and the FT remains valid.

\section{EFFECT OF NONCYCLIC EVOLUTIONS}

Adiabatic non-cyclic geometric (ANG) phases 5,6 arise when the adiabatic parametrization takes place in a noncyclic way, i.e. the curve traced in the parameter space is not closed. It has been experimentally observed in the evolution of spatial degrees of freedom of neutrons using interferometry ${ }^{62}$. Equation (7) is a general expression for the acquired geometric contribution due to adiabatic modulation of two parameters. When the contour $\mathcal{C}$ is cyclic, we arrived at Eq. (8), the PB contribution. In principle, geometric nature of Eq. (77) can be extended to cases when $\mathcal{C}$ is noncyclic and leads to the noncyclic adiabatic contributions which also has a geometric interpretation 63,64 .

Equation (6) is equivalent to (appendix):

$$
\left.|\rho(t)\rangle\rangle=\sum_{m=+,-} a_{m}(0)\left|R_{m}(t)\right\rangle\right\rangle e^{-\int_{0}^{t} d t^{\prime} \chi_{m}\left(t^{\prime}\right)-\zeta_{m}\left(t^{\prime}\right)}(, 45)
$$

where $\chi_{m}\left(t^{\prime}\right)=\left\langle\left\langle L_{m}\left(t^{\prime}\right) \mid \dot{R}_{m}\left(t^{\prime}\right\rangle\right\rangle\right.$ is the diagonal element $(m=+,-)$ of the matrix $B_{d}\left(t^{\prime}\right)$ in Eq. (6). Equation (45) is simply an expansion of $|\rho(t)\rangle\rangle$ in terms of the right eigenvector of $\hat{\mathcal{L}}(t)$ with the initial expansion coefficient $a_{m}(0)$. Similarly, $\langle\langle\rho(t)|$ can be expanded in terms of the left eigenvectors, $\left\langle\left\langle L_{m}(t)\right.\right.$ such that $\langle\langle\rho(0)|=$ $\sum_{m= \pm} b_{m}(0)\left\langle\left\langle L_{m}(t)\right|\right.$. As discussed in Sec.(III), we can write,

$$
\int_{0}^{t} d t^{\prime} \chi_{m}(t)=\int_{\mathcal{C}} \chi_{m}(\mathbf{x}) \cdot d \mathbf{x},
$$

where $\mathcal{C}$ is now an open contour in the parameter space, $\mathbf{x}$. Note that, unlike for the case of cyclic driving with closed contour $\mathcal{C}$, for noncyclic evolution, $\int_{\mathcal{C}} \chi_{m}(\mathbf{x}) \cdot d \mathbf{x}$ is not gauge invariant as we discuss below. In order to extract a gauge invariant geometric contribution we project Eq.(45) with the initial density vector $\langle\langle\rho(0)|$ to obtain

$$
\langle\langle\rho(0) \mid \rho(t)\rangle\rangle=\sum_{m= \pm} \Xi_{m}(t) \exp \left\{\int_{0}^{t} d t^{\prime} \zeta_{m}\left(t^{\prime}\right)\right\},
$$

where,

$$
\Xi_{m}(t)=a_{m}(0)\left\langle\left\langle\rho(0) \mid R_{m}(t)\right\rangle\right\rangle e^{-\int_{\mathcal{C}} \chi_{m}(\mathbf{x}) \cdot d \mathbf{x}} .
$$

$\Xi_{m}(t)$ is independent of parametrization of path $\mathcal{C}$ in the parameter space. $\Xi_{m}(t)$ is also invariant under a local gauge transformation, $\left.\left.\left|R_{m}(t)\right\rangle\right\rangle \rightarrow \exp (i \eta(t))\left|R_{m}(t)\right\rangle\right\rangle$, $\left\langle\left\langle L_{m}(t)\right| \rightarrow\left\langle\left\langle L_{m}(t)\right| \exp \{-i \eta(t)\}\right.\right.$ where $\eta(t)$ is an arbitrary differentiable function. Both these factors together guarantee the geometric nature $e^{63,65}$ of $\Xi_{m}(t)$. The geometric nature of phases during noncyclic evolutions has also been shown by closing the open contour using geodesics and parallel transport law arguments ${ }^{6}$. Equation (48) is the general expression for an adiabatic, noncyclic geometric contribution (ANG) which reduces to Eq. (77) for a cyclic driving over the closed contour C. Note that, although, the integral in Eq. (48) is reparametrization independent but it is not gauge invariant and hence not an observable for open contour $\mathcal{C}$.

For an arbitrary driving, in the long time limit, only $m=+$ term dominates. The steady state density matrix is given by $\left.\left|\rho_{s}(t)\right\rangle\right\rangle=\{\beta(t) / \Gamma, \alpha(t) / \Gamma\}$ and $a_{m}(0)$ is obtained by solving $\left.|\rho(0)\rangle\rangle=\sum_{m= \pm} a_{m}(0)\left|R_{m}(0)\right\rangle\right\rangle$. So,

$$
\begin{aligned}
a_{+}(0)\left\langle\left\langle\rho(0) \mid R_{+}(t)\right\rangle\right\rangle & =\frac{\alpha(0)^{2}+\beta(0)^{2}}{\Gamma^{2} \alpha(0) \alpha(t)} \\
& \times\left(\alpha(0) \alpha(t)+\beta(0) \beta(t)^{2}\right), \\
\chi_{+}(t) & =-\frac{d}{d t} \ln \frac{\alpha(t)}{\alpha(0)},
\end{aligned}
$$

Substituting Eqs.(49) and (50) in (48), we find for the generalized ANG contribution

$$
\Xi_{+}(t)=\frac{\alpha(0)^{2}+\beta(0)^{2}}{\Gamma^{2} \alpha(t)^{2}}(\alpha(0) \alpha(t)+\beta(0) \beta(t)),
$$

for an arbitrary modulation of the thermodynamic equilibrium of the leads. For noncyclic driving, $t \neq t_{p}$, the 
evolution of the density matrix is influenced by the geometric contribution and therefore, unlike the cyclic case, the flux in the junction is also affected by the geometric (ANG) part.

Taking a time-derivative in Eq. (6), we get,

$$
\left.|\dot{\rho}(t)\rangle\rangle=\left(\hat{\mathcal{L}}_{d}(t)+\hat{\mathcal{L}}_{g}(t)\right)|\rho(t)\rangle\right\rangle
$$

where $\hat{\mathcal{L}}_{d(g)}(t)$ is the dynamic (geometric) Liouvillian given by

$$
\begin{aligned}
& \hat{\mathcal{L}}_{d}(t)=U(t) \Lambda(t) U^{-1}(t) \\
& \hat{\mathcal{L}}_{g}(t)=\dot{U}(t) U^{-1}(t) .
\end{aligned}
$$

The steady state electronic flux between the system and left lead is defined as $j(t)=e\left\langle\left\langle\hat{N}\left|\hat{\mathcal{L}}^{(l)}(t)\right| \rho_{s}(t)\right\rangle\right\}^{66}$, where $\hat{N}=\{1,0\}$ and $\hat{\mathcal{L}}^{(l)}(t)$ is the Liouvillian containing only the terms from the left lead and $\left.\left|\rho_{s}(t)\right\rangle\right\rangle$ is the steady state density matrix. Following Eq. (52), we can split, $\hat{\mathcal{L}}^{(l)}(t)=\hat{\mathcal{L}}_{d}^{(l)}(t)+\hat{\mathcal{L}}_{g}^{(l)}(t)$, corresponding to the dynamic and the geometric Liouvillians. Since there exists a time dependent driving, the steady state is changing with respect to the external driving. At each instant of driving steady state is well defined. So the flux, $j(t)$, is time dependent and represents the flux at each instant in time during the adiabatic change. The dynamic contribution is given by,

$$
j_{d}(t)=I_{o}^{S N}\left(f_{l}(t)-f_{r}(t)\right),
$$

where $I_{o}^{S N}=2 e \Gamma_{l} \Gamma_{r} / \Gamma$ is the steady state current in absence of driving in the shot noise limit $(T=0 K)$. The geometric (ANG) contribution is,

$$
\begin{aligned}
j_{g}(t) & =e\left\langle\left\langle\hat{N}\left|\hat{\mathcal{L}}_{g}^{(l)}(t)\right| \rho_{s}(t)\right\rangle\right\rangle \\
& =e \sum_{m= \pm}\left\langle\left\langle\hat{N}\left|\hat{\mathcal{L}}_{g}^{(l)}(t)\right| R_{m}(0)\right\rangle\right\rangle\left\langle\left\langle L_{m}(0) \mid \rho_{s}(t)\right\rangle\right\rangle\langle
\end{aligned}
$$

Here, in the second line, we have used the resolution of unity in terms of $\left.\left|R_{m}(t)\right\rangle\right\rangle$ and $\left\langle\left\langle L_{m}(t)\right|\right.$. Since, $\left\langle\left\langle L_{-}(t) \mid \rho_{s}(t)\right\rangle\right\rangle=0$, we can write,

$$
j_{g}(t)=e\left\langle\left\langle\hat{N}\left|\hat{\mathcal{L}}_{g}^{(l)}(t)\right| R_{+}(0)\right\rangle\right\rangle\left\langle\left\langle L_{+}(0) \mid \rho_{s}(t)\right\rangle\right\rangle .
$$

Using $\left\langle\langle\rho(0)|=\sum_{m= \pm} b_{m}(0)\left\langle\left\langle L_{m}(0)\right|\right.\right.$, we get

$$
j_{g}(t)=\frac{e}{b_{+}(0)}\left\langle\left\langle\hat{N}\left|\hat{\mathcal{L}}_{g}^{(l)}(t)\right| R_{+}(0)\right\rangle\right\rangle\left\langle\left\langle\rho(0) \mid \rho_{s}(t)\right\rangle\right\rangle,
$$

where, $\left\langle\left\langle\rho(0) \mid \rho_{s}(t)\right\rangle\right\rangle$ is given by Eq. (47) at the steady state and contains information about the ANG contribution as given by Eq. (51). Following Eq. (59), we can substitute the initial and the steady state values and write down the geometric flux as,

$$
j_{g}(t)=\frac{e \beta(t)}{\Gamma} \chi_{+}^{(l)}(t),
$$

where $\chi_{+}^{(l)}(t)$ is given by Eq. (50) with only the left lead's contribution. For cyclic driving, $\chi_{+}(t)=0$, since $t$ is an integral multiple of of $t_{p}$ giving $j_{g}(t)=0$.
We can define average dynamic flux per measurement time, $\mathcal{T}$ as

$$
\begin{aligned}
\left\langle j_{d}\right\rangle & =\frac{1}{\mathcal{T}} \int_{0}^{\mathcal{T}} j_{d}(t) d t \\
& =I_{o}^{S N} \frac{e^{-R}-1}{\mathcal{T}} \int_{0}^{\mathcal{T}} f_{r}(t)\left(1-f_{l}(t)\right) d t,
\end{aligned}
$$

where, $R$ is defined by Eq. (43) and acts as thermodynamic force that drives the flux. For the chosen sinusoidal drivings, Eq. (62) becomes,

$$
\left\langle j_{d}\right\rangle=I_{o}\left(1-\frac{m^{2}}{2}\right)+I_{o}^{S N} \frac{m^{2}}{n \pi} f(n, \phi),
$$

with,

$f(n, \phi)=-\frac{1}{4}\left[f_{l} \sin (2 n \pi)-f_{r}\{\sin (2 n \pi+2 \phi)-\sin (2 \phi)\}\right]$,

and $I_{o}=I_{o}^{S N}\left(f_{l}-f_{r}\right)$, is the steady state current in the absence of driving. Here, we have used, $\mathcal{T}=n t_{p}$. For the cyclic case, $n$ is an integer which represents the number of cycles during the driving and $f(n, \phi)=0$. For the noncyclic case, $0<n<1$ and $f(n, \phi) \neq 0$.

The average geometric flux in a measurement window can be written as,

$$
\begin{aligned}
\left\langle j_{g}\right\rangle & =\frac{1}{n t_{p}} \int_{0}^{n t_{p}} j_{g}(t) d t \\
& =-\frac{e}{n t_{p} \Gamma} \int_{0}^{n t_{p}} \frac{\beta(t) \dot{\alpha}_{l}(t)}{\alpha_{l}(t)} d t .
\end{aligned}
$$

For the chosen sinusoidal drivings, we get

$$
\begin{aligned}
\left\langle j_{g}\right\rangle= & -\frac{e}{n t_{p} \Gamma}\left\{\Delta\left[\beta\left(n t_{p}\right) \log \left(\tilde{f}_{l}\left(n t_{p}\right)\right)\right]+2 m^{2} \Gamma_{r} f_{r}\{\right. \\
& \left.\left.f_{l} \cos ^{2}(\phi) \Delta\left[\tilde{f}_{l}\left(n t_{p}\right) \log \left(f_{l}\left(n t_{p}\right)\right)\right]+k \sin (2 \phi) A_{n}\right\}\right\}
\end{aligned}
$$

where,

$$
\begin{aligned}
A_{n} & =\sin \left(2 k n t_{p}\right)\left\{4 \sqrt{\tilde{f}_{l}} \sqrt{\tilde{f}_{l}(0)} \arctan \left(\frac{\sqrt{\tilde{f}_{l} \tan \left(k n t_{p}\right)}}{\sqrt{\tilde{f}_{l}(0)}}\right)\right. \\
& \left.-2 k n t_{p}\left(\tilde{f}_{l}+\tilde{f}_{l}(0)\right)-m^{2} f_{l}\left(\log \left(\tilde{f}_{l}\left(n t_{p}\right)\right)-1\right)\right\} \cdot(68)
\end{aligned}
$$

Here we have used $\Delta\left[X\left(n t_{p}\right)\right]=X\left(n t_{p}\right)-X(0)$. Equation (67) vanishes for both $n \in \mathbb{Z}$ and $m=0$. Note that $\left\langle j_{g}\right\rangle \neq 0$ when $\phi=0$. This tells us that for noncyclic evolutions, even if the system parameters are identically driven, there is always a geometric contribution to the total flux. We show the behavior of $\left\langle j_{g}\right\rangle$ in Fig.(77) as a function of $n$ for two different time-periods. As can be seen, the flux gradually increases from $n=0$ and reaches a maximum and then goes to zero as $n$ approaches 1 (cyclic case). 


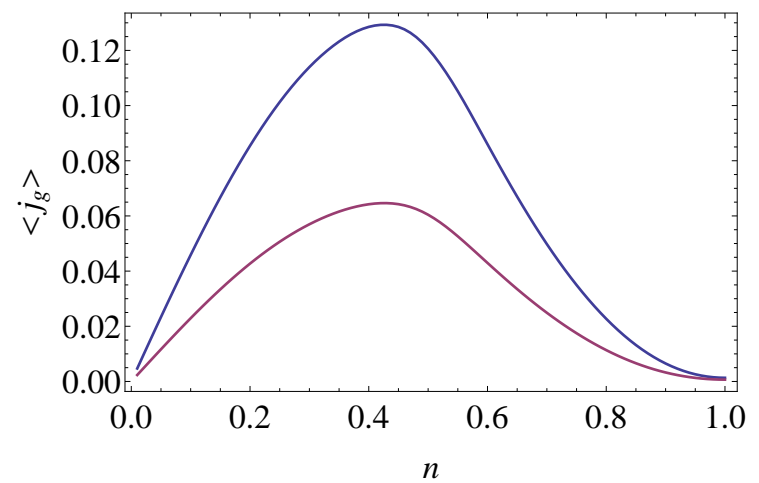

FIG. 7. The average geometric flux (Eq. (67)) as a function of $n$. The upper (lower) curve is simulated for $t_{p}=10 \pi(5 \pi)$. It is zero at $n=0,1$. Simulation parameters used are $m=$ $0.9, f_{l}=0.9, f_{r}=0.1, \Gamma_{l}=1.25, \Gamma_{r}=0.75, \phi=\pi / 4$.

\section{CONCLUSION}

We have analyzed the effects of cyclic (PB) and noncyclic (ANG) geometric contributions in an adiabatically driven current carrying quantum junction. Time evolution of the reduced density matrix for a resonant level is shown to be unaffected by the $\mathrm{PB}$ contribution arising due to periodic, adiabatic driving. A generating function formalism was used to compute the statistics of the net electron transfered through the junction. The PB contribution to the first cumulant is found to be zero. However the higher order cumulants (fluctuations) were affected by the PB contribution. We derived analytic expressions for the geometric contributions to fluctuations. The phase difference between the drivings could be tuned to alter the statistics of the net electron-transfer from antibunched to bunched. We also observed that the fluctuation theorem (or GC symmetry) is violated in presence of a nonzero geometric (PB or ANG) contribution. However, we recover a driven steady-state FT when the geometric contribution vanishes. In this case, we identified a thermodynamic force and showed that the GC symmetry is preserved.

In case of noncyclic evolutions, the geometric contribution (ANG) affects the density matrix of the system which in turn influences the net flux across the junction. Unlike the PB contribution, ANG contribution is nonzero even when the phase difference between the drivings is zero.

We note that, performing a similar analysis for the case when, instead of the Fermi functions, couplings $\Gamma_{l}$ and $\Gamma_{r}$ are modulated periodically in time, the PB curvature vanishes altogether in the QME framework, preserving the FT. This happens because the coupling induced lifetime is neglected in the QME analysis, which is essential to observe geometric effects due to such modulations. Such effects are usually incorporated using non-equilibrium Greens function technique $\underline{\underline{67}}$. We also observe that in single electron counting measurements, where one keeps track of only the incoming or outgoing electron transfer processes between the leads and system, the geometric curvature is zero.

Acknowledgments: HPG acknowledges the financial support from University Grants Commission, New Delhi under the Senior Research Fellowship Scheme. HPG also thanks Ross H. Mckenzie and Hari K. Yadalam for interesting discussions. BKA thanks the hospitality from Indian Institute of Science, Bangalore. UH acknowledges the support from Indian Institute of Science, Bangalore.

\section{Appendix A}

Derivation of the Pancharatnam-Berry generating function: We can re-write Eq. (10) as

$$
\begin{aligned}
\frac{\partial \rho(\tau)}{\partial \tau} & =\alpha(\tau) \hat{c}_{s} \rho(\tau) \hat{c}_{s}^{\dagger}-\beta(\tau) \rho(\tau) \hat{c}_{s} \hat{c}_{s}^{\dagger} \\
& -\alpha(\tau) \hat{c}_{s}^{\dagger} \hat{c}_{s} \rho(\tau)+\beta(\tau) \hat{c}_{s}^{\dagger} \rho(\tau) \hat{c}_{s},
\end{aligned}
$$

where $\alpha(\tau)=\alpha_{l}(\tau)+\alpha_{r}(\tau)$ and $\beta(\tau)=\beta_{l}(\tau)+\beta_{r}(\tau)$ are the system to leads and leads to system electron transfer rates respectively,

$$
\begin{aligned}
& \alpha_{X}(\tau)=\Gamma_{X}\left(1-f_{X}\left(\epsilon_{s}, \tau\right)\right), \\
& \beta_{X}(\tau)=\Gamma_{X} f_{X}\left(\epsilon_{s}, \tau\right) .
\end{aligned}
$$

A quantum master equation for such a system is $|\dot{\rho}(t)\rangle\rangle=\hat{\mathcal{L}}(t)|\rho(t)\rangle\rangle,|\rho(t)\rangle\rangle=\left\{\rho_{11}, \rho_{00}\right\}$ is the reduced density vector for the system containing the population only. $\hat{\mathcal{L}}$ is the Liouvillian in the many body space. We do not include the coherences $\left(\rho_{01}, \rho_{10}\right)$ because they exponentially die out and are decoupled from populations.

In this driven case, we can obtain the eigenbasis (Eq.(3)) as,

$$
\Lambda(\tau)=\Lambda(0)=\left(\begin{array}{cc}
0 & 0 \\
0 & -2\left(\Gamma_{l}+\Gamma_{r}\right)
\end{array}\right)
$$

and $U(\tau)$, that diagonalizes $\hat{\mathcal{L}}(t)$, is given by,

$$
U(\tau)=\left(\begin{array}{cc}
\frac{\beta(\tau)}{\alpha(\tau)} & -1 \\
1 & 1
\end{array}\right),
$$

The term, $B_{d}(\tau)=\operatorname{diag}\left[U^{-1}(\tau) \dot{U}(\tau)\right]$ which when integrated over a time-period, $t=t_{p}=\pi / k$ is zero. Also $\nabla \times B_{d}\left(f_{l}, f_{r}\right)=0$. This indicates the field is conservative and leaves the dynamics of the density matrix unaffected as it doesn't acquire any geometric contribution during its time evolution.

To quantify the statistics of electron transfer, we start by defining a moment generating function, $G(\lambda, t)$ for the PDF corresponding to the net number of particles, $q$ transferred between left lead and system. The equation of motion for $G(\lambda, t)$ is

$$
\dot{G}(\lambda, t)=\langle\langle\mathbf{1}|M(\lambda, t)| \rho(\lambda, t)\rangle\rangle .
$$


$M(\lambda, t)$ is the characteristic counting Liouvillian. We have denoted the time and counting-field dependent density vector as $|\rho(\lambda, t)\rangle\rangle$. We can expand it in the basis of the right eigenvector of $M(\lambda, t)$ with time dependent expansion coefficients $a_{n}(t) \stackrel{19}{\underline{t}}$,

$$
\left.|\rho(\lambda, t\rangle\rangle=\sum_{n= \pm} a_{n}(t) e^{\int_{0}^{t} \zeta_{n}\left(\lambda, t^{\prime}\right) d t^{\prime}}\left|R_{n}(\lambda, t)\right\rangle\right\rangle
$$

The instantaneous eigen values, $\zeta_{ \pm}(\lambda, t)$ of $M(\lambda, t)$ matrix can be written as

$$
\begin{aligned}
\zeta_{ \pm}(\lambda, t) & =-\alpha(t)-\beta(t) \\
& \pm \sqrt{(\alpha(t)-\beta(t))^{2}+4\left(\alpha_{\lambda}(t) \beta_{\lambda}(t)\right)} .
\end{aligned}
$$

Here $\zeta_{+}\left(\zeta_{-}\right)$is the smaller (larger eigenvalue) with $\alpha_{\lambda}(\tau)=\alpha_{l}(\tau) e^{-\lambda}+\alpha_{r}(\tau)$ and $\beta_{\lambda}(\tau)=\beta_{l}(\tau) e^{\lambda}+\beta_{r}(\tau)$. Substituting Eq. (A7) in Eq.(13), time evolution of the expansion coefficients can be written as, we get,

$$
\begin{aligned}
\left.\sum_{n} \dot{a}_{n}(t) e^{\int_{0}^{t} \zeta_{n}\left(\lambda, t^{\prime}\right) d t^{\prime}}\left|R_{n}(\lambda, t)\right\rangle\right\rangle & \\
= & \left.-\sum_{n} a_{n}(t) e^{\int_{0}^{t} \zeta_{n}\left(\lambda, t^{\prime}\right) d t^{\prime}}\left|\dot{R}_{n}(\lambda, t)\right\rangle\right\rangle .
\end{aligned}
$$

Left multiplying by $\left\langle\left\langle L_{m}(\lambda, t \mid\right.\right.$ and using $\left\langle\left\langle L_{m}\left(\lambda, t\left|R_{n}(\lambda, t\rangle\right\rangle=\delta_{m n}\right.\right.\right.$ gives,

$$
\begin{aligned}
\dot{a}_{m}(t) & =-a_{m}(t)\left\langle\left\langle L_{m}(\lambda, t) \mid \dot{R}_{m}(\lambda, t)\right\rangle\right\rangle \\
& -\sum_{m \neq n} a_{n}(t) e^{\int_{0}^{t}\left(\zeta_{n}\left(\lambda, t^{\prime}\right)-\zeta_{m}\left(\lambda, t^{\prime}\right)\right) d t^{\prime}} \\
& \times\left\langle\left\langle L_{m}(\lambda, t) \mid \dot{R}_{n}(\lambda, t\rangle\right\rangle .\right.
\end{aligned}
$$

Left and right eigenvectors together form an orthonormal set. In the adiabatic limit, since the eigenstates of the system do not mix, the inner product of the time derivative of the right eigen vector and the left eigenvector corresponding to different eigenvalues vanishes, i.e $\left\langle\left\langle L_{m}(\lambda, t) \mid \dot{R}_{n}(\lambda, t)\right\rangle\right\rangle=0$. The solution of Eq. A10 can now be written down as,

$$
a_{m}(t)=a_{m}(0) \exp \left\{-\int_{0}^{t} d t^{\prime}\left\langle\left\langle L_{m}\left(\lambda, t^{\prime}\right) \mid \dot{R}_{m}\left(\lambda, t^{\prime}\right\rangle\right\rangle\right\} .\right.
$$

Substituting Eq. A11 in Eq. A77, we get

$$
\begin{aligned}
|\rho(\lambda, t\rangle\rangle & \left.=\sum_{m=+,-} a_{m}(0)\left|R_{m}(\lambda, t)\right\rangle\right\rangle \\
& \times \exp \left\{-\int_{0}^{t} d t^{\prime}\left\langle\left\langle L_{m}\left(\lambda, t^{\prime}\right) \mid \dot{R}_{m}\left(\lambda, t^{\prime}\right\rangle\right\rangle-\zeta_{m}\left(\lambda, t^{\prime}\right)\right\} .\right.
\end{aligned}
$$

The generating function is given by the trace of the counting density-matrix, $G(\lambda, t)=\langle\langle\mathbf{1} \mid \rho(\lambda, t)\rangle\rangle$. So,

$$
\begin{aligned}
G(\lambda, t) & =\sum_{m=+,-} a_{m}(0)\left\langle\left\langle\mathbf{1} \mid R_{m}(\lambda, t)\right\rangle\right\rangle \\
& \times \exp \left\{-\int_{0}^{t} d t^{\prime}\left\langle\left\langle L_{m}\left(\lambda, t^{\prime}\right) \mid \dot{R}_{m}\left(\lambda, t^{\prime}\right\rangle\right\rangle-\zeta_{m}(\lambda, t)\right\},\right.
\end{aligned}
$$

where the left and the right eigenvectors of $M(\lambda)$ are obtained as,

$$
\begin{aligned}
\left.\left|R_{ \pm}(\lambda, t)\right\rangle\right\rangle & =\left\{u_{ \pm}(t), 1\right\} \\
\left\langle\left\langle L_{ \pm}(\lambda, t)\right|\right. & =\frac{1}{u_{+}(t)-u_{-}(t)}\left\{ \pm 1, \mp u_{\mp}(t)\right\},
\end{aligned}
$$

with,

$$
u_{ \pm}(t)=\frac{-(\alpha(t)-\beta(t)) \pm \sqrt{(\alpha(t)-\beta(t))^{2}+4 \alpha_{\lambda}(t) \beta_{\lambda}(t)}}{2 \alpha_{\lambda}(t)} .
$$

We denote the time period of evolution by $t_{p}$ and assume that the total measurement time can be expressed as multiple of the periodic modulation and write $t=n t_{p}$, when $n>>1, n \in \Re$. So we get,

$$
\begin{aligned}
G(\lambda, t) & =-\sum_{m=+,-} a_{m}(0)\left\langle\left\langle\mathbf{1} \mid R_{m}\left(\lambda, n t_{p}\right)\right\rangle\right\rangle \\
& \times n \int_{0}^{t_{p}} d t^{\prime}\left[\left\langle\left\langle L_{m}\left(\lambda, t^{\prime}\right) \mid \dot{R}_{m}\left(\lambda, t^{\prime}\right\rangle\right\rangle-\zeta_{m}\left(\lambda, t^{\prime}\right)\right]\right.
\end{aligned}
$$

At long times, the contribution from the eigenvalue,$\zeta_{-}$ is exponentially suppressed. Hence, at large times,

$$
\begin{aligned}
G(\lambda, t) & \approx a_{+}(0)\left\langle\left\langle\mathbf{1} \mid R_{+}(\lambda, t)\right\rangle\right\rangle \\
& e^{\frac{t}{t_{p}} \int_{0}^{t_{p}}\left(\zeta_{+}\left(\lambda, t^{\prime}\right)-\left\langle\left\langle L_{+}\left(\lambda, t^{\prime}\left|\dot{R}_{+}\left(\lambda, t^{\prime}\right\rangle\right\rangle\right) d t^{\prime}\right.\right.\right.} .
\end{aligned}
$$

At the steady state, it is more convenient to work with the scaled cumulant generating function defined as

$$
\begin{aligned}
S(\lambda) & =\lim _{t \rightarrow \infty} \frac{1}{t} \ln G(\lambda, t) \\
& =\lim _{n \rightarrow \infty} \frac{1}{n t_{p}}\left[\ln a_{+}(0)\left\langle\left\langle\mathbf{1} \mid R_{+}\left(\lambda, n t_{p}\right)\right\rangle\right\rangle\right] \\
& +\frac{1}{t_{p}} \int_{0}^{t_{p}} \zeta_{+}\left(\lambda, t^{\prime}\right) d t^{\prime} \\
& -\frac{1}{t_{p}} \int_{0}^{t_{p}}\left\langle\left\langle L_{+}\left(\lambda, t^{\prime}\right) \mid \dot{R}_{+}\left(\lambda, t^{\prime}\right)\right\rangle\right\rangle d t^{\prime} .
\end{aligned}
$$

The first term in Eq. A20 is constant and goes to zero when $n$ is an integer and $n \rightarrow \infty$, since $\left.\left.\left|R_{+}\left(\lambda, n t_{p}\right)\right\rangle\right\rangle=\left|R_{+}(\lambda, 0)\right\rangle\right\rangle$. For non integer, $n$, $\left.\left.\left|R_{+}\left(\lambda, n t_{p}\right) \neq\right| R_{+}(\lambda, 0)\right\rangle\right\rangle$, the term will survive.

So, for integer values on $n$, the scaled cumulant gener- ating function can be expressed as a sum of a dynamic $\left(S_{d}(\lambda)\right)$ and geometric $\left(S_{g}(\lambda)\right)$ scaled cumulant generating functions given by,

$$
S(\lambda)=S_{d}(\lambda)+S_{g}(\lambda)
$$

and

$$
\begin{aligned}
& S_{d}(\lambda)=\frac{1}{t_{p}} \int_{0}^{t_{p}} \zeta_{+}\left(\lambda, t^{\prime}\right) d t^{\prime}, \\
& S_{g}(\lambda)=\frac{-1}{t_{p}} \int_{0}^{t_{p}}\left\langle\left\langle L_{+}\left(\lambda, t^{\prime}\right) \mid \dot{R}_{+}\left(\lambda, t^{\prime}\right)\right\rangle\right\rangle d t^{\prime} .
\end{aligned}
$$


The first and second $\lambda$ derivatives of Eq. A21 evaluated at $\lambda=0$ give the flux and steady state fluctuation. Replacing the line integral as a contour integral we recover Eq. (19).

We now proceed to derive Eqs. (20) and (21), i.e express the Berry potential, $\left\langle\left\langle L_{+}(\lambda, \mathbf{x})\left|\partial_{\mathbf{x}}\right| R_{+}(\lambda, \mathbf{x})\right\rangle\right\rangle$ in terms of the parameter derivatives of the counting Liouvillian $M(\lambda)$. Here, $\mathbf{x}$ is a vector with two parameters $x$ and $y$. For the right eigenvector of $\hat{M}(\lambda)$ corresponding to the $i$-th eigenvalue $(i=+,-)$ we have:

$$
\begin{aligned}
\hat{M}(\lambda)\left|R_{i}(\lambda, t\rangle\right\rangle & \left.=\zeta_{i}\left|R_{i}(\lambda, t)\right\rangle\right\rangle \\
\left.\Longrightarrow \partial_{y} \hat{M}(\lambda)\left|R_{i}(\lambda, t)\right\rangle\right\rangle & \left.=\partial_{y} \zeta_{i}\left|R_{i}(\lambda, t)\right\rangle\right\rangle \\
\Longrightarrow \partial_{y}\left(\hat{M}(\lambda)-\zeta_{i}\right)\left|R_{i}(\lambda, t\rangle\right\rangle & \left.=\left(\zeta_{i}-\hat{M}(\lambda)\right)\left|\partial_{y} R_{i}(\lambda, t)\right\rangle\right\rangle .
\end{aligned}
$$

Taking the projection with the left eigenvector $\left\langle\left\langle L_{j}(\lambda, t)\right|\right.$, we get

$$
\left\langle\left\langle L_{j}(\lambda, t) \mid \partial_{y} R_{i}(\lambda, t)\right\rangle\right\rangle=\frac{\left\langle\left\langle L_{j}(\lambda, t)\left|\partial_{y} \hat{M}(\lambda)\right| R_{i}(\lambda, t)\right\rangle\right\rangle}{\left(\zeta_{i}-\zeta_{j}\right)^{2}} .
$$

Similarly steps can be done with the left eigenvector and taking projection with $\left.\left|R_{j}(\lambda, t)\right\rangle\right\rangle$, we get

$$
\left\langle\left\langle\partial_{x} L_{i}(\lambda, t) \mid R_{j}(\lambda, t)\right\rangle\right\rangle=\frac{\left\langle\left\langle L_{i}(\lambda, t)\left|\partial_{x} \hat{M}(\lambda)\right| R_{j}(\lambda, t)\right\rangle\right\rangle}{\left(\zeta_{i}-\zeta_{j}\right)^{2}} .
$$

The Pancharatnam-Berry potential in the two parameter vector space $\mathbf{x}$ is given by $\left\langle\left\langle L_{+}(\lambda, \mathbf{x})\left|\partial_{\mathbf{x}}\right| R_{+}(\lambda, \mathbf{x})\right\rangle\right\rangle$, whose curl gives the curvature, $\mathfrak{B}^{\lambda}(x, y)$,

$$
\mathfrak{B}^{\lambda}(x, y)=\left\langle\left\langle\partial_{x} L_{+}(\lambda) \mid \partial_{y} R_{+}(\lambda)\right\rangle\right\rangle-\left\langle\left\langle\partial_{y} L_{+}(\lambda) \mid \partial_{x} R_{+}(\lambda)\right\rangle\right\rangle .
$$

Substituting Eq. A27 and Eq. A28 in Eq. A29 and using $\left.\sum_{i= \pm}\left|R_{i}(\lambda, t)\right\rangle\right\rangle\left\langle\left\langle L_{i}(\lambda, t)\right|=\mathbf{1}\right.$, we can recover Eq. (21).

Evaluation of Contour area, $C_{A}$ :

The parametric dependence on time $\tau$ for the Fermi functions can be recast as an equation of ellipse. Let $f_{l}(\tau)$ and $f_{r}(\tau)$ represent the time dependent Fermi-functions such that

$$
\begin{aligned}
& f_{l}(\tau)=f_{l}\left(1-m^{2} \cos ^{2}(k \tau)\right) \\
& f_{r}(\tau)=f_{r}\left(1-m^{2} \cos ^{2}(k \tau+\phi)\right) .
\end{aligned}
$$

Here, $0<m<1$, and $\phi$ is the phase difference. These two equations can be recast as a single ellipse equation of the form, $A f_{l}(\tau)^{2}+B f_{r}^{2}(\tau)+C f_{l}(\tau) f_{r}(\tau)+D f_{l}(\tau)+$ $E f_{r}(\tau)+F=0$, where,

$$
\begin{aligned}
& A=\frac{1}{f_{l}^{2}} \\
& B=\frac{1}{f_{r}^{2}} \\
& C=\frac{-2 \cos (2 \phi)}{f_{l} f_{r}} \\
& D=\frac{\left(m^{2}-2\right)}{f_{l}}\left(\sin ^{2}(2 \phi)-2 \sin ^{2}(\phi) \cos (2 \phi)\right), \\
& E=\frac{m^{2}-2}{f_{r}} \sin ^{2}(\phi), \\
& F=\left(1-m^{2}\right) \sin ^{2}(2 \phi)+\left(2-m^{2}\right)^{2} \sin ^{4}(\phi) .
\end{aligned}
$$

Here, $C^{2}<4 A B$ preserving the ellipse at all times. The centers of the ellipse is at $\left\{x_{o}, y_{0}\right\}$ given by

$$
\begin{aligned}
& x_{o}=\frac{E C-2 B D}{4 A B-C^{2}}, \\
& y_{o}=\frac{D C-2 A E}{4 A B-C^{2}} .
\end{aligned}
$$

The major $\left(a_{M}\right)$ and minor $\left(a_{m}\right)$ axes are given by

$$
\begin{aligned}
& a_{M}=\frac{-F_{o}}{A \cos ^{2}\left(\theta_{R}\right)+B \sin ^{2}\left(\theta_{R}\right)-0.5 \sin ^{2}\left(\theta_{R}\right)},(A \\
& a_{m}=\frac{-F_{o}}{A \sin ^{2}\left(\theta_{R}\right)+B \cos ^{2}\left(\theta_{R}\right)-0.5 \sin ^{2}\left(\theta_{R}\right)},(A
\end{aligned}
$$

where

$$
F_{o}=C x_{o} y_{o}+D x_{o}+E y_{o}+A x_{o}^{2}+B y_{o}^{2}+F,(
$$

and $\theta_{R}$ is the angle of rotation of the ellipse given by

$$
\theta_{R}=\frac{1}{2} \arctan \left(\frac{C}{A-B}\right)
$$

The contour area is given by $C_{A}=\oiint f_{l} f_{r}=\pi a_{M} a_{m}$. For $\phi=0, D=E=F=0$ and as a consequence, $C_{A}=0$.
1 David Jeffery Griffiths, Introduction to quantum mechanics (Pearson Education India, 2005).

2 Arno Bohm, Ali Mostafazadeh, Hiroyasu Koizumi, Qian Niu, and Josef Zwanziger, The Geometric Phase in Quantum Systems: Foundations, Mathematical Concepts, and Applications in Molecular and Condensed Matter Physics
(Springer Science \& Business Media, 2013).

3 Shivaramakrishnan Pancharatnam, "Generalized theory of interference, and its applications. part i. coherent pencils," in Proceedings of the Indian Academy of Sciences, Section A, Vol. 44 (Indian Academy of Sciences, 1956) pp. 247-262.

4 Michael V Berry, "Quantal phase factors accompanying 
adiabatic changes," in Proc. R. Soc. London, Ser. A, Vol. 392 (The Royal Society, 1984) pp. 45-57.

5 Arun Kumar Pati, "Geometric aspects of noncyclic quantum evolutions," Phys. Rev. A 52, 2576 (1995).

6 Joseph Samuel and Rajendra Bhandari, "General setting for berry's phase," Phys. Rev. Lett. 60, 2339-2342 (1988)

7 J Anandan, "Non-adiabatic non-abelian geometric phase," Phys. Lett. A 133, 171-175 (1988).

8 Yakir Aharonov and J Anandan, "Phase change during a cyclic quantum evolution," Phys. Rev. Lett. 58, 1593 (1987).

9 Jan C Budich, Dietrich G Rothe, Ewelina M Hankiewicz, and Björn Trauzettel, "All-electric qubit control in heavy hole quantum dots via non-abelian geometric phases," Phys. Rev. B 85, 205425 (2012).

10 L-M Duan, JI Cirac, and P Zoller, "Geometric manipulation of trapped ions for quantum computation," Science 292, 1695-1697 (2001).

11 Y Taguchi, Y Oohara, H Yoshizawa, N Nagaosa, and Y Tokura, "Spin chirality, berry phase, and anomalous hall effect in a frustrated ferromagnet," Science 291, 2573-2576 (2001).

12 Yuanbo Zhang, Yan-Wen Tan, Horst L Stormer, and Philip Kim, "Experimental observation of the quantum hall effect and berry's phase in graphene," Nature 438, 201-204 (2005).

13 PJ Leek, JM Fink, A Blais, R Bianchetti, M Göppl, JM Gambetta, DI Schuster, L Frunzio, RJ Schoelkopf, and A Wallraff, "Observation of berry's phase in a solid-state qubit," Science 318, 1889-1892 (2007).

14 Di Xiao, Ming-Che Chang, and Qian Niu, "Berry phase effects on electronic properties," Rev. Mod. Phys. 82, 1959 (2010).

15 Jing-Tao Lu, Mads Brandbyge, and Per Hedegård, "Blowing the fuse: Berrys phase and runaway vibrations in molecular conductors," Nano Lett. 10, 1657-1663 (2010).

16 Angelo Carollo, I Fuentes-Guridi, M Franca Santos, and Vlatko Vedral, "Geometric phase in open systems," Phys. Rev. Lett. 90, 160402 (2003).

17 MS Sarandy and DA Lidar, "Abelian and non-abelian geometric phases in adiabatic open quantum systems," Phys. Rev. A 73, 062101 (2006).

18 Dario Calvani, Alessandro Cuccoli, Nikitas I Gidopoulos, and Paola Verrucchi, "Open quantum systems and the parametric representation: From entanglement to berrys phase," Int. J. Theor. Phys. 53, 3434-3446 (2014).

19 Jie Ren, Peter Hänggi, Baowen Li, et al., "Berry-phaseinduced heat pumping and its impact on the fluctuation theorem," Phys. Rev. Lett. 104, 170601 (2010).

20 Shijie $\mathrm{Hu}$, Ari $\mathrm{M}$ Turner, Karlo Penc, Frank Pollmann, et al., "Berry-phase-induced dimerization in onedimensional quadrupolar systems," Phys. Rev. Lett. 113, 027202 (2014).

21 Huan-Qiang Zhou, Sam Young Cho, and Ross H. McKenzie, "Gauge fields, geometric phases, and quantum adiabatic pumps," Phys. Rev. Lett. 91, 186803 (2003)

22 Mark A Reed, C Zhou, CJ Muller, TP Burgin, and JM Tour, "Conductance of a molecular junction," Science 278, 252-254 (1997).

23 Cancan Huang, Alexander V Rudnev, Wenjing Hong, and Thomas Wandlowski, "Break junction under electrochemical gating: testbed for single-molecule electronics," Chemical Society Reviews 44, 889-901 (2015).

24 Ronnie Kosloff and Amikam Levy, "Quantum heat engines and refrigerators: Continuous devices," Annu. Rev. Phys. Chem 65, 365-93 (2014).

25 Himangshu Prabal Goswami and Upendra Harbola, "Thermodynamics of quantum heat engines," Phys. Rev. A 88, 013842 (2013).

${ }^{26}$ Gaël Reecht, Fabrice Scheurer, Virginie Speisser, Yannick J Dappe, Fabrice Mathevet, and Guillaume Schull, "Electroluminescence of a polythiophene molecular wire suspended between a metallic surface and the tip of a scanning tunneling microscope," Phys. Rev. Lett. 112, 047403 (2014).

27 Sriharsha V Aradhya and Latha Venkataraman, "Singlemolecule junctions beyond electronic transport," Nature nanotechnology 8, 399-410 (2013).

28 Hyunwook Song, Mark A Reed, and Takhee Lee, "Single molecule electronic devices," Adv. Mater. 23, 1583-1608 (2011).

29 Lanlan Sun, Yuri A Diaz-Fernandez, Tina A Gschneidtner, Fredrik Westerlund, Samuel Lara-Avila, and Kasper Moth-Poulsen, "Single-molecule electronics: from chemical design to functional devices," Chem. Soc. Rev. 43, 73787411 (2014).

30 Toshimasa Fujisawa, Toshiaki Hayashi, Ritsuya Tomita, and Yoshiro Hirayama, "Bidirectional counting of single electrons," Science 312, 1634-1636 (2006).

31 Upendra Harbola, Bijay Kumar Agarwalla, and Shaul Mukamel, "Frequency-domain stimulated and spontaneous light emission signals at molecular junctions," J. Chem. Phys. 141, 074107 (2014).

32 Himangshu Prabal Goswami, Weijie Hua, Yu Zhang, Shaul Mukamel, and Upendra Harbola, "Electroluminescence in molecular junctions: a diagrammatic approach," J. Chem. Theory Comput. 11, 4304-4315 (2015).

33 Tamar Shamai and Yoram Selzer, "Spectroscopy of molecular junctions," Chem. Soc. Rev. 40, 2293-2305 (2011).

34 Jaesuk Hwang, Martin Pototschnig, Robert Lettow, Gert Zumofen, Alois Renn, Stephan Götzinger, and Vahid Sandoghdar, "A single-molecule optical transistor," Nature 460, 76-80 (2009).

35 PW Brouwer, "Scattering approach to parametric pumping," Phys. Rev. B 58, R10135 (1998).

36 M Switkes, CM Marcus, K Campman, and AC Gossard, "An adiabatic quantum electron pump," Science 283, 1905-1908 (1999).

37 Gabriel González and Michael N Leuenberger, "Berryphase blockade in single-molecule magnets," Phys. Rev. Lett. 98, 256804 (2007).

38 Upendra Harbola and Shaul Mukamel, "Superoperator nonequilibrium greens function theory of many-body systems; applications to charge transfer and transport in open junctions," Phys. Rep. 465, 191-222 (2008).

39 Abhishek Dhar, "Heat transport in low-dimensional systems," Adv. Phys. 57, 457-537 (2008).

40 Daniel R Ward, David A Corley, James M Tour, and Douglas Natelson, "Vibrational and electronic heating in nanoscale junctions," Nat. Nanotechnol. 6, 33-38 (2011).

41 DA Bagrets and Yu V Nazarov, "Full counting statistics of charge transfer in coulomb blockade systems," Phys. Rev. B 67, 085316 (2003).

42 Abraham Nitzan and Mark A Ratner, "Electron transport in molecular wire junctions," Science 300, 1384-1389 (2003).

43 Massimiliano Esposito, Upendra Harbola, and Shaul Mukamel, "Nonequilibrium fluctuations, fluctuation the- 
orems, and counting statistics in quantum systems," Rev. Mod. Phys. 81, 1665 (2009).

${ }^{44}$ G Gallavotti and EGD Cohen, "Dynamical ensembles in nonequilibrium statistical mechanics," Phys. Rev. Lett. 74, 2694 (1995).

45 Saito Keiji and Dhar Abhishek, "Fluctuation theorem in quantum heat conduction," Phys. Rev. Lett. 99, 180601 (2007).

46 Tameem Albash, Sergio Boixo, Daniel A Lidar, and Paolo Zanardi, "Quantum adiabatic markovian master equations," New J. Phys. 14, 123016 (2012).

47 Lorenzo Campos Venuti, Tameem Albash, Daniel A. Lidar, and Paolo Zanardi, "Adiabaticity in open quantum systems," Phys. Rev. A 93, 032118 (2016)

48 Massimiliano Esposito, Upendra Harbola, and Shaul Mukamel, "Fluctuation theorem for counting statistics in electron transport through quantum junctions," Phys. Rev. B 75, 155316 (2007).

49 NA Sinitsyn and Ilya Nemenman, "The berry phase and the pump flux in stochastic chemical kinetics," EPL (Europhysics Letters) 77, 58001 (2007).

50 Tatsuro Yuge, Takahiro Sagawa, Ayumu Sugita, and Hisao Hayakawa, "Geometrical pumping in quantum transport: Quantum master equation approach," Phys. Rev. B 86, 235308 (2012).

${ }^{51}$ Ugo Fano, "Ionization yield of radiations. ii. the fluctuations of the number of ions," Physical Review 72, 26 (1947).

52 David Roxbee Cox and Peter Adrian Walter Lewis, The statistical analysis of series of events (John Wiley and Sons, 1966).

53 Mathias Albert, Géraldine Haack, Christian Flindt, and Markus Büttiker, "Electron waiting times in mesoscopic conductors," Phys. Rev. Lett. 108, 186806 (2012).

54 MP Silverman, "On the feasibility of observing electron antibunching in a field-emission beam," Phys. Lett. A 120, 442-446 (1987).

${ }^{55}$ M Iannuzzi, A Orecchini, F Sacchetti, P Facchi, and S Pascazio, "Direct experimental evidence of free-fermion antibunching," Phys. Rev. Lett. 96, 080402 (2006).

56 O Zarchin, M Zaffalon, M Heiblum, D Mahalu, and
V Umansky, "Two-electron bunching in transport through a quantum dot induced by kondo correlations," Phys. Rev. B 77, 241303 (2008).

57 O Zarchin, YC Chung, M Heiblum, D Rohrlich, and V Umansky, "Electron bunching in transport through quantum dots in a high magnetic field," Phys. Rev. Lett. 98, 066801 (2007).

58 Hugo Touchette, "The large deviation approach to statistical mechanics," Phys. Rep. 478, 1-69 (2009).

59 SR Srinivasa Varadhan, "Asymptotic probabilities and differential equations," Commun. Pure Appl. Math. 19, 261286 (1966).

60 Steven A Orszag and CM Bender, Advanced mathematical methods for scientists and engineers (Mac Graw Hill, 1978).

61 Massimiliano Esposito, Upendra Harbola, and Shaul Mukamel, "Entropy fluctuation theorems in driven open systems: Application to electron counting statistics," Phys. Rev. E 76, 031132 (2007).

62 Stefan Filipp, Yuji Hasegawa, Rudolf Loidl, and Helmut Rauch, "Noncyclic geometric phase due to spatial evolution in a neutron interferometer," Phys. Rev. A 72, 021602 (2005)

63 N Mukunda and R Simon, "Quantum kinematic approach to the geometric phase. i. general formalism," Annals of Physics 228, 205-268 (1993).

64 Erik Sjöqvist, Arun K Pati, Artur Ekert, Jeeva S Anandan, Marie Ericsson, Daniel KL Oi, and Vlatko Vedral, "Geometric phases for mixed states in interferometry," Phys. Rev. Lett. 85, 2845 (2000).

65 Gonzalo Garcia de Polavieja and Erik Sjoqvist, "Extending the quantal adiabatic theorem: Geometry of noncyclic motio," Am. J. Phys. 66, 431-438 (1998).

66 Upendra Harbola, Massimiliano Esposito, and Shaul Mukamel, "Quantum master equation for electron transport through quantum dots and single molecules," Phys. Rev. B 74, 235309 (2006).

67 Hari Kumar Yadalam and Upendra Harbola, "Statistics of an adiabatic charge pump," Phys. Rev. B 93, 035312 (2016). 